\title{
FUZZY CONTROL FOR INPUT CONSTRAINED PASSIVE AFFINE TAKAGI- SUGENO FUZZY MODELS: AN APPLICATION TO TRUCK-TRAILER SYSTEM
}

\author{
Wen-Jer Chang \\ Department of Marine Engineering, National Taiwan Ocean University, Keelung, Taiwan, R.O.C., \\ wjchang@mail.ntou.edu.tw \\ Cheung-Chieh Ku \\ Department of Electrical Engineering, National Taiwan Ocean University, Keelung, Taiwan, R.O.C. \\ Chien-Hsun Huang \\ Department of Marine Engineering, National Taiwan Ocean University, Keelung, Taiwan, R.O.C.
}

Follow this and additional works at: https://jmstt.ntou.edu.tw/journal

Part of the Engineering Commons

\section{Recommended Citation}

Chang, Wen-Jer; Ku, Cheung-Chieh; and Huang, Chien-Hsun (2011) "FUZZY CONTROL FOR INPUT CONSTRAINED PASSIVE AFFINE TAKAGI-SUGENO FUZZY MODELS: AN APPLICATION TO TRUCK-TRAILER SYSTEM," Journal of Marine Science and Technology. Vol. 19: Iss. 5, Article 3.

DOI: $10.51400 / 2709-6998.2161$

Available at: https://jmstt.ntou.edu.tw/journal/vol19/iss5/3

This Research Article is brought to you for free and open access by Journal of Marine Science and Technology. It has been accepted for inclusion in Journal of Marine Science and Technology by an authorized editor of Journal of Marine Science and Technology. 


\section{FUZZY CONTROL FOR INPUT CONSTRAINED PASSIVE AFFINE TAKAGI-SUGENO}

FUZZY MODELS: AN APPLICATION TO TRUCK-TRAILER SYSTEM

\section{Acknowledgements}

The authors would like to express their sincere gratitude to anonymous reviewers who gave us some constructive comments, criticisms and suggestions. This work was supported by the National Science Council of the Republic of China under Contract NSC97-2221-E-019-023. 


\title{
FUZZY CONTROL FOR INPUT CONSTRAINED PASSIVE AFFINE TAKAGI-SUGENO FUZZY MODELS: AN APPLICATION TO TRUCK-TRAILER SYSTEM
}

\author{
Wen-Jer Chang*, Cheung-Chieh $\mathrm{Ku}^{* *}$, and Chien-Hsun Huang*
}

Key words: input constraint, affine Takagi-Sugeno fuzzy model, passive property, Iterative Linear Matrix Inequality.

\begin{abstract}
This paper describes a design of fuzzy controller for the input constrained discrete nonlinear passive systems via affine Takagi-Sugeno (T-S) fuzzy models. The stability conditions are derived based on Lyapunov and passive theories for the closed-loop system. The fuzzy controller design is accomplished by using the concept of Parallel Distribution Compensation (PDC). According to the proposed fuzzy control approach, the controlled system can achieve passive property and the input constraint, simultaneously. The Iterative Linear Matrix Inequality (ILMI) algorithm is applied in this paper to find the feasible solutions for the proposed fuzzy controller design approach. At last, a numerical simulation to the trucktrailer system is provided to demonstrate the applicability and effectiveness of the proposed fuzzy control methodology.
\end{abstract}

\section{INTRODUCTION}

The passivity theory $[4,9,11-15,20,21]$ provides a nice tool for analyzing the stability of the nonlinear or linear systems by using an input-output description based on energyrelated consideration. The main ideal behind this is that many important physical systems have certain input-output properties related to the conservation, dissipation and transport of energy. By constructing proper Lyapunov function and using some analytic techniques, several sufficient conditions are given to ensure the passivity of the closed-loop systems. As for a storage function, it measures the amount for energy stored in the internal of the system, so it is naturally described

Paper submitted 07/03/09; revised 09/21/09; accepted 05/21/10. Author for correspondence: Wen-Jer Chang (e-mail: wjchang@mail.ntou.edu.tw).

*Department of Marine Engineering, National Taiwan Ocean University, Keelung, Taiwan, R.O.C.

**Department of Electrical Engineering, National Taiwan Ocean University, Keelung, Taiwan, R.O.C. by using internal variables of system, that it, state variables. Passivity theory is intimately related to Lyapunov stability theory. The Lyapunov function is represented as a quadratic function of state variables in this paper. The researchers and engineers can design controllers to achieve passivity properties for the systems based on Lyapunov function. The passive property has many types for expressing total energy and input energy of systems via power supply. The choice of the power supply is a very interesting task for the control systems $[4,11$, $12,14,20,21]$.

The T-S fuzzy model $[2,3,5-8,10,16-19]$ provides an effective representation of complex nonlinear systems in terms of fuzzy sets and fuzzy reasoning applied to a set of linear input/output subsystems. One can approach the trajectory of an original nonlinear system via combination of the membership function and the sublinear systems of the T-S fuzzy model. Hence, the T-S fuzzy model becomes a useful approach to investigate the nonlinear systems. The T-S fuzzy model is described by IF-THEN rules and the corresponding fuzzy controller design is developed based on PDC technique [2, 3, 5-8, 10, 16-19]. The pioneers, Kim and his colleagues [5] have provided a sufficient condition for the stability analysis of the affine T-S fuzzy models in sense of Lyapunov inequality. The affine T-S fuzzy model means the T-S fuzzy model of which consequent part is affine and which has a constant bias term. The affine T-S fuzzy model is more natural and appealing to human beings than the homogeneous one. The homogeneous T-S fuzzy model is usually studied by the researchers due to the ease of analysis [5-7, 16-19].

In this paper, both the controller design synthesis of discrete-time affine T-S fuzzy systems and control input constraint discussed in [18] are considered. In general, the stability conditions of affine T-S fuzzy systems are most Bilinear Matrix Inequality (BMI) problems which were discussed in $[2,3,8,10]$. The BMI problem is difficult to solve by numerically convex optimization technique and cannot be calculated via LMI toolbox in MATLAB. For this reason, it is necessary to take the LMI form to replace the BMI form via some transform techniques and relation functions. In synthesis, the derived stability conditions and the controller design 
problem can be numerically solved by an iterative manner, which is called as ILMI algorithm [2, 3, 8, 10]. The ILMI algorithm is developed to solve the BMI problem; hence, it can be used to find the feasible solutions of fuzzy controller design problems for the affine T-S fuzzy models. Extending the ILMI algorithm developed in [2, 3, 8, 10], a modified ILMI algorithm is derived in this paper to find suitable fuzzy controllers such that the control input constraint and passive property of the affine T-S fuzzy models can be achieved. Hence, the contribution of this paper is to develop a useful fuzzy controller design methodology to deal with the input constrained control problem for the discrete affine T-S fuzzy models that achieve passive performance constraints.

The principle structure of this paper is described as follows. The descriptions of discrete affine T-S fuzzy model and passive property are presented in Section 2. Based on the PDC concept, the stability analysis and input constrained fuzzy controller design synthesis are solved for discrete affine T-S fuzzy model in Section 3. Applying the proposed fuzzy controller design approach to the truck-trailer system via numerical simulations is shown in Section 4. Finally, concluding remarks are made in Section 5.

\section{SYSTEM DESCRIPTIONS AND STABILITY ANALYSIS WITH PASSIVITY}

The passivity and Lyapunov stability conditions of discrete affine T-S fuzzy systems are discussed via Lyapunov criterion in this section. Besides, the $S$-procedure is used to derive the Lyapunov stability conditions with passivity constraints. The detail of $S$-procedure can be referred to $[1,2,10]$. By using the IF-THEN fuzzy rule form, the discrete-time affine T-S fuzzy system is described as follows.

\section{Plant Part:}

Rule i: IF $x_{1}(k)$ is $M_{i 1}$ and $x_{2}(k)$ is $M_{i 2}$ and ... and $x_{\mathrm{p}}(k)$ is $M_{\mathrm{ip}}$

$$
\begin{gathered}
\text { THEN } x(k+1)=\mathbf{A}_{\mathrm{i}} x(k)+\mathbf{B}_{u \mathrm{i}} u(k)+\mathbf{B}_{w \mathrm{i}} w(k)+\mathbf{a}_{\mathrm{i}} \\
y(k)=\mathbf{C}_{\mathrm{i}} x(k)+\mathbf{D}_{\mathrm{i}} w(k) \quad \mathrm{i}=1, \ldots, \mathrm{r}
\end{gathered}
$$

where $\mathbf{A}_{\mathrm{i}} \in \mathfrak{R}^{\mathrm{n}_{x} \times \mathrm{n}_{x}}, \mathbf{B}_{u \mathrm{i}} \in \mathfrak{R}^{\mathrm{n}_{x} \times \mathrm{n}_{u}}, \mathbf{B}_{w \mathrm{i}} \in \mathfrak{R}^{\mathrm{n}_{x} \times \mathrm{n}_{y}}, \mathbf{a}_{\mathrm{i}} \in \mathfrak{R}^{\mathrm{n}_{x}}, \mathbf{C}_{\mathrm{i}} \in$ $\mathfrak{R}^{\mathrm{n}_{y} \times \mathrm{n}_{x}}$, and $\mathbf{D}_{\mathrm{i}} \in \mathfrak{R}^{\mathrm{n}_{y} \times \mathrm{n}_{y}}$ are constant matrices and $x(k) \in \mathfrak{R}^{\mathrm{n}_{x}}$ is the state vector, $y(k) \in \mathfrak{R}^{\mathrm{n}_{y}}$ is the output vector, $w(k) \in \mathfrak{R}^{\mathrm{n}_{y}}$ is the square-integrable exogenous input vector, $u(k) \in \mathfrak{R}^{\mathrm{n}_{u}}$ is the control input vector, $M_{\mathrm{ij}}$ is the fuzzy set, $\mathrm{p}$ is the premise variable number and $\mathrm{r}$ is number of fuzzy model rules. Given the pair of $(x(k), u(k))$, the closed-loop of the fuzzy model (1) can be referred as follows.

$$
x(k+1)=\frac{\sum_{\mathrm{i}=1}^{\mathrm{r}} \omega_{\mathrm{i}}(x(k))\left(\mathbf{A}_{\mathrm{i}} x(k)+\mathbf{B}_{u \mathrm{i}} u(k)+\mathbf{B}_{w \mathrm{i}} w(k)+\mathbf{a}_{\mathrm{i}}\right)}{\sum_{\mathrm{i}=1}^{\mathrm{r}} \omega_{\mathrm{i}}(x(k))}
$$

$$
y(k)=\frac{\sum_{\mathrm{i}=1}^{\mathrm{r}} \omega_{\mathrm{i}}(x(k))\left(\mathbf{C}_{\mathrm{i}} x(k)+\mathbf{D}_{\mathrm{i}} w(k)\right)}{\sum_{\mathrm{i}=1}^{\mathrm{r}} \omega_{\mathrm{i}}(x(k))} \mathrm{i}=1, \ldots, \mathrm{r}
$$

or

$$
\begin{gathered}
x(k+1)=\sum_{\mathrm{i}=1}^{\mathrm{r}} h_{\mathrm{i}}(x(k))\left(\mathbf{A}_{\mathrm{i}} x(k)+\mathbf{B}_{u i} u(k)+\mathbf{B}_{w \mathrm{i}} w(k)+\mathbf{a}_{\mathrm{i}}\right) \\
y(k)=\sum_{\mathrm{i}=1}^{\mathrm{r}} h_{\mathrm{i}}(x(k))\left(\mathbf{C}_{\mathrm{i}} x(k)+\mathbf{D}_{\mathrm{i}} w(k)\right)
\end{gathered}
$$

where

$$
\begin{aligned}
& \omega_{\mathrm{i}}(x(k))=\prod_{\mathrm{j}=1}^{\mathrm{p}} M_{\mathrm{ij}}\left(x_{\mathrm{j}}(k)\right) \\
& h_{\mathrm{i}}(x(k))=\frac{\omega_{\mathrm{i}}(x(k))}{\sum_{\mathrm{i}=1}^{\mathrm{r}} \omega_{\mathrm{i}}(x(k))}
\end{aligned}
$$

and $M_{\mathrm{ij}}\left(x_{\mathrm{j}}(k)\right)$ is the grade of membership function of $x_{\mathrm{j}}(k)$ in $M_{\mathrm{ij}}$. It is assumed that $\omega_{\mathrm{i}}(x(k)) \geq 0, \sum_{\mathrm{i}=1}^{\mathrm{r}} \omega_{\mathrm{i}}(x(k))>0, h_{\mathrm{i}}(x(k)) \geq$ $0, \sum_{\mathrm{i}=1}^{\mathrm{r}} h_{\mathrm{i}}(x(k))=1$ and $\mathrm{i}=1, \ldots, \mathrm{r}$ for all $x(k)$

For the nonlinear plant provided in (2) or (3), the fuzzy controller is designed to share the same fuzzy set with the plant. This fuzzy controller is developed based on the PDC technique that can be referred to $[2,3,5-8,10,16-19]$. The PDC-based fuzzy controller has the following form.

\section{Controller Part:}

Rule i: IF $x_{1}(k)$ is $M_{\mathrm{i} 1}$ and $x_{2}(k)$ is $M_{\mathrm{i} 2}$ and $\ldots$ and $x_{\mathrm{p}}(k)$ is $M_{\mathrm{ip}}$

$$
\text { THEN } u(k)=-\mathbf{F}_{\mathrm{i}} x(k), \quad \mathrm{i}=1, \ldots, \mathrm{r}
$$

Based on PDC concept, the fuzzy controller can be represented via summation as follows.

$$
u(k)=\sum_{\mathrm{i}=1}^{\mathrm{r}} h_{\mathrm{i}}(x(k))\left(-\mathbf{F}_{\mathrm{i}} x(k)\right) \quad \mathrm{i}=1, \ldots, \mathrm{r}
$$

Substituting (7) into (3a), one can obtain the corresponding closed-loop system such as

$$
\begin{aligned}
x(k+1)= & \sum_{\mathrm{i}=1}^{\mathrm{r}} \sum_{\mathrm{j}=1}^{\mathrm{r}} h_{\mathrm{i}}(x(k)) h_{\mathrm{j}}(x(k))\left(\mathbf{A}_{\mathrm{i}} x(k)-\mathbf{B}_{u \mathrm{i}} \mathbf{F}_{\mathrm{j}} x(k)\right) \\
& \left.+\mathbf{B}_{w \mathrm{i}} w(k)+\mathbf{a}_{\mathrm{i}}\right)
\end{aligned}
$$




$$
\begin{aligned}
& =\sum_{\mathrm{i}=1}^{\mathrm{r}} \sum_{\mathrm{j}=1}^{\mathrm{r}} h_{\mathrm{i}}(x(k)) h_{\mathrm{j}}(x(k))\left(\left(\mathbf{A}_{\mathrm{i}}-\mathbf{B}_{u \mathrm{i}} \mathbf{F}_{\mathrm{j}}\right) x(k)+\mathbf{B}_{w \mathrm{i}} w(k)+\mathbf{a}_{\mathrm{i}}\right) \\
& =2 \sum_{\substack{\mathrm{i}=1 \\
\mathrm{i}<\mathrm{j})}}^{\mathrm{r}} \sum_{\mathrm{j}=1}^{\mathrm{r}} h_{\mathrm{i}}(x(k)) h_{\mathrm{j}}(x(k))\left(\left(\frac{\left(\mathbf{A}_{\mathrm{i}}-\mathbf{B}_{u \mathbf{i}} \mathbf{F}_{\mathrm{j}}\right)+\left(\mathbf{A}_{\mathrm{j}}-\mathbf{B}_{u \mathrm{j}} \mathbf{F}_{\mathrm{i}}\right)}{2}\right) x(k)\right. \\
& \left.+\left(\frac{\mathbf{B}_{w \mathrm{i}}+\mathbf{B}_{w \mathrm{j}}}{2}\right) w(k)+\frac{\mathbf{a}_{\mathrm{i}}+\mathbf{a}_{\mathrm{j}}}{2}\right)+\sum_{\substack{\mathrm{i}=1 \\
(\mathrm{i}=\mathrm{j})}}^{\mathrm{r}} h_{\mathrm{i}}^{2}(x(k))\left(\left(\mathbf{A}_{\mathrm{i}}-\mathbf{B}_{u \mathrm{i}} \mathbf{F}_{\mathrm{i}}\right)\right. \\
& \left.\times x(k)+\mathbf{B}_{w \mathrm{i}} w(k)+\mathbf{a}_{\mathrm{i}}\right) \\
& =\sum_{\mathrm{i}=1}^{\mathrm{r}} \sum_{\mathrm{j}=1}^{\mathrm{r}} h_{\mathrm{i}}(x(k)) h_{\mathrm{j}}(x(k))\left(\left(\frac{\left(\mathbf{A}_{\mathrm{i}}-\mathbf{B}_{u \mathrm{i}} \mathbf{F}_{\mathrm{j}}\right)+\left(\mathbf{A}_{\mathrm{j}}-\mathbf{B}_{u \mathrm{j}} \mathbf{F}_{\mathrm{i}}\right)}{2}\right)\right. \\
& \left.\times x(k)+\left(\frac{\mathbf{B}_{w \mathrm{i}}+\mathbf{B}_{w \mathrm{j}}}{2}\right) w(k)+\frac{\mathbf{a}_{\mathrm{i}}+\mathbf{a}_{\mathrm{j}}}{2}\right) \\
& \equiv \sum_{\mathrm{i}=1}^{\mathrm{r}} \sum_{\mathrm{j}=1}^{\mathrm{r}} h_{\mathrm{i}}(x(k)) h_{\mathrm{j}}(x(k))\left(\mathbf{G}_{\mathrm{ij}} x(k)+\mathbf{N}_{\mathrm{ij}} w(k)+\tilde{\mathbf{a}}_{\mathrm{ij}}\right)
\end{aligned}
$$

where $\mathbf{G}_{\mathrm{ij}}=\frac{\left(\mathbf{A}_{\mathrm{i}}-\mathbf{B}_{u \mathrm{i}} \mathbf{F}_{\mathrm{j}}\right)+\left(\mathbf{A}_{\mathrm{j}}-\mathbf{B}_{u \mathrm{j}} \mathbf{F}_{\mathrm{i}}\right)}{2}, \quad \mathbf{N}_{\mathrm{ij}}=\frac{\mathbf{B}_{w \mathrm{i}}+\mathbf{B}_{w \mathrm{j}}}{2}$ and $\tilde{\mathbf{a}}_{\mathrm{ij}}=\frac{\mathbf{a}_{\mathrm{i}}+\mathbf{a}_{\mathrm{j}}}{2}$.

\section{Assumption 1:}

The region $\mathrm{X}_{\mathrm{i}} \subseteq \mathfrak{R}^{\mathrm{n}_{x}}$ is $\mathrm{n}_{x}$-dimensional convex polyhedron which is called as a cell. The cell indices are denoted by $\tilde{I}$ and union of all cells $\mathrm{X} \subseteq \bigcup_{\mathrm{i} \in \tilde{I}} \mathrm{X}_{\mathrm{i}}$ is referred to as the partition.

Let $\hat{I} \subseteq \tilde{I}$ be the set of indices for the fuzzy rules that contain the origin and others be the set of indices for the fuzzy rules that not contain the origin. Here, the origin $x(0)=0$ is the equilibrium point of the discrete affine T-S fuzzy model and it is assumed $\mathbf{a}_{\mathrm{i}}=0$ for $\mathrm{i} \in \hat{I}$.

The concept of the passive property proposes a very useful tool in the stability analysis and controller design synthesis for linear or nonlinear dynamic systems. The strictly input passive property is introduced in the following definition that will be used to discuss the passivity constraints for the discrete affine T-S fuzzy systems in the paper.

\section{Definition 1 [4]:}

The system (3) with exogenous input $w(k)$ and output $y(k)$ is said to be strictly input passive if

$$
2 \sum_{k=0}^{k_{\mathrm{q}}} y^{\mathrm{T}}(k) w(k)>\sum_{k=0}^{k_{\mathrm{q}}} \gamma w^{\mathrm{T}}(k) w(k)
$$

where $\gamma \geq 0, k_{\mathrm{q}}>0$ and $x(0)=0$.

There are many other types of passive inequality, which can be referred to the references $[13,20,21]$. The passivity analysis problem can also be considered as an $H_{\infty}$ analysis problem via some assumptions that can be refereed to $[11,15$, 21]. The passivity conditions for the discrete-time affine T-S fuzzy system (8) and (3b) can be characterized by the following theorem.

\section{Theorem 1:}

If there exist a positive definite matrix $\mathbf{P}>0$, scalars $\gamma \geq 0$ and $\varepsilon_{\mathrm{ijq}} \geq 0$ for satisfying the following conditions, then the discrete-time affine T-S fuzzy model (3) driven by fuzzy controller (7) is strictly input passive and Lyapunov stable.

$$
\left[\begin{array}{ll}
\boldsymbol{\Phi}_{11} & \boldsymbol{\Phi}_{21}^{\mathrm{T}} \\
\boldsymbol{\Phi}_{21} & \boldsymbol{\Phi}_{22}
\end{array}\right]<0, \quad \mathrm{i}, \mathrm{j} \in \hat{I}
$$

$\left[\begin{array}{ccc}\boldsymbol{\Phi}_{11}-\sum_{\mathrm{q}=1}^{\mathrm{p}} \varepsilon_{\mathrm{ijq}} \mathbf{T}_{\mathrm{ijq}} & \boldsymbol{\Phi}_{21}^{\mathrm{T}} & \mathbf{G}_{\mathrm{ij}}^{\mathrm{T}} \mathbf{P} \tilde{\mathbf{a}}_{\mathrm{ij}}-\sum_{\mathrm{q}=1}^{\mathrm{p}} \varepsilon_{\mathrm{ijq}} \mathbf{n}_{\mathrm{ijq}} \\ \boldsymbol{\Phi}_{21} & \boldsymbol{\Phi}_{22} & \mathbf{N}_{\mathrm{ij}}^{\mathrm{T}} \mathbf{P} \tilde{\mathbf{a}}_{\mathrm{ij}} \\ \tilde{\mathbf{a}}_{\mathrm{ij}}^{\mathrm{T}} \mathbf{P} \mathbf{G}_{\mathrm{ij}}-\sum_{\mathrm{q}=1}^{\mathrm{p}} \varepsilon_{\mathrm{ijq}} \mathbf{n}_{\mathrm{ijq}}^{\mathrm{T}} & \tilde{\mathbf{a}}_{\mathrm{ij}}^{\mathrm{T}} \mathbf{P} \mathbf{N}_{\mathrm{ij}} & \tilde{\mathbf{a}}_{\mathrm{ij}}^{\mathrm{T}} \mathbf{P} \tilde{\mathbf{a}}_{\mathrm{ij}}-\sum_{\mathrm{q}=1}^{\mathrm{p}} \varepsilon_{\mathrm{ijq}} v_{\mathrm{ijq}}\end{array}\right]<0, \mathrm{i}, \mathrm{j} \notin \hat{I}$

where

$$
\begin{gathered}
\boldsymbol{\Phi}_{11}=\mathbf{G}_{\mathrm{ij}}^{\mathrm{T}} \mathbf{P} \mathbf{G}_{\mathrm{ij}}-\mathbf{P} \\
\boldsymbol{\Phi}_{21}=\mathbf{N}_{\mathrm{ij}}^{\mathrm{T}} \mathbf{P} \mathbf{G}_{\mathrm{ij}}-\mathbf{C}_{\mathrm{i}} \\
\boldsymbol{\Phi}_{22}=\mathbf{N}_{\mathrm{ij}}^{\mathrm{T}} \mathbf{P} \mathbf{N}_{\mathrm{ij}}-\mathbf{D}_{\mathrm{i}}-\mathbf{D}_{\mathrm{i}}^{\mathrm{T}}+\gamma \mathbf{I}
\end{gathered}
$$

and $\mathbf{T}_{\mathrm{ijq}} \in \mathfrak{R}^{\mathrm{n}_{x} \times \mathrm{n}_{x}}, \mathbf{n}_{\mathrm{ijq}} \in \mathfrak{R}^{\mathrm{n}_{x}}$ and $v_{\mathrm{ijq}} \in \mathfrak{R}$ are defined such as

$$
\Theta_{\mathrm{ijq}}(x(k))=x^{\mathrm{T}}(k) \mathbf{T}_{\mathrm{ijq}} x(k)+2 x^{\mathrm{T}}(k) \mathbf{n}_{\mathrm{ijq}}+v_{\mathrm{ijq}} \leq 0
$$

\section{Proof:}

First, we select the case of fuzzy rules $\mathrm{i} \notin \hat{I}$ in following proof. Use the storage function $V(x(k))=x^{T}(k) \mathbf{P} x(k)$ as a Lyapunov function for the closed-loop system (8). By evaluating the first forward difference of $V(x(k))$ along the trajectories of (8), one has

$$
\begin{aligned}
& \Delta V(x(k))=x^{\mathrm{T}}(k+1) \mathbf{P} x(k+1)-x^{\mathrm{T}}(k) \mathbf{P} x(k) \\
& =\sum_{\mathrm{i}=1}^{\mathrm{r}} \sum_{\mathrm{j}=1}^{\mathrm{r}} \sum_{\mathrm{s}=1}^{\mathrm{r}} \sum_{\mathrm{z}=1}^{\mathrm{r}} h_{\mathrm{i}}(x(k)) h_{\mathrm{j}}(x(k)) h_{\mathrm{s}}(x(k)) h_{\mathrm{z}}(x(k))
\end{aligned}
$$




$$
\begin{aligned}
& \times\left(\mathbf{G}_{\mathrm{ij}} x(k)+\mathbf{N}_{\mathrm{ij}} w(k)+\tilde{\mathbf{a}}_{\mathrm{ij}}\right)^{\mathrm{T}} \mathbf{P}\left(\mathbf{G}_{\mathrm{sz}} x(k)+\mathbf{N}_{\mathrm{sz}} w(k)+\tilde{\mathbf{a}}_{\mathrm{sz}}\right) \\
& -x^{\mathrm{T}}(k) \mathbf{P} x(k)
\end{aligned}
$$

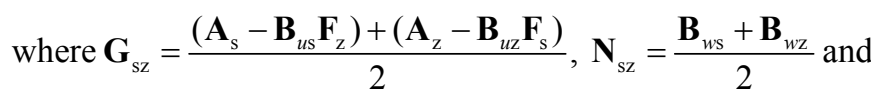
$\tilde{\mathbf{a}}_{\mathrm{sz}}=\frac{\mathbf{a}_{\mathrm{s}}+\mathbf{a}_{\mathrm{z}}}{2}$.

Choosing the performance function such as

$$
J_{D}=\sum_{k=0}^{k_{\mathrm{q}}}\left(\gamma w^{\mathrm{T}}(k) w(k)-2 y^{\mathrm{T}}(k) w(k)\right)
$$

with zero initial condition for all $w(k)$. Then, for any nonzero $w(k)$ one has

$$
\begin{aligned}
J_{D} & \leq \sum_{k=0}^{k_{\mathrm{q}}}\left(\gamma w^{\mathrm{T}}(k) w(k)-2 y^{\mathrm{T}}(k) w(k)\right)+V(x(k)) \\
& =\sum_{k=0}^{k_{\mathrm{q}}}\left(\gamma w^{\mathrm{T}}(k) w(k)-2 y^{\mathrm{T}}(k) w(k)\right)+\sum_{k=0}^{k_{\mathrm{q}}} \Delta V(x(k)) \\
& =\sum_{k=0}^{k_{\mathrm{q}}}\left(\gamma w^{\mathrm{T}}(k) w(k)-2 y^{\mathrm{T}}(k) w(k)+\Delta V(x(k))\right) \\
& \equiv \sum_{k=0}^{k_{\mathrm{q}}} L(x, w, k)
\end{aligned}
$$

According to (17) and (18), one has

$$
\begin{aligned}
L(x, w, k) & =\Delta V(x(k))+\gamma w^{\mathrm{T}}(k) w(k)-2 y^{\mathrm{T}}(k) w(k) \\
& \leq \sum_{\mathrm{i}=1}^{\mathrm{r}} \sum_{\mathrm{j}=1}^{\mathrm{r}} h_{\mathrm{i}}(x(k)) h_{\mathrm{j}}(x(k))\left[\begin{array}{c}
x(k) \\
w(k) \\
1
\end{array}\right]^{\mathrm{T}} \\
& \times\left[\begin{array}{ccc}
\boldsymbol{\Phi}_{11} & \boldsymbol{\Phi}_{21}^{\mathrm{T}} & \mathbf{G}_{\mathrm{ij}}^{\mathrm{T}} \mathbf{P} \tilde{\mathbf{a}}_{\mathrm{ij}} \\
\boldsymbol{\Phi}_{21} & \boldsymbol{\Phi}_{22} & \mathbf{N}_{\mathrm{ij}}^{\mathrm{T}} \mathbf{P} \tilde{\mathbf{a}}_{\mathrm{ij}} \\
\tilde{\mathbf{a}}_{\mathrm{ij}}^{\mathrm{T}} \mathbf{P} \mathbf{G}_{\mathrm{ij}} & \tilde{\mathbf{a}}_{\mathrm{ij}}^{\mathrm{T}} \mathbf{P} \mathbf{N}_{\mathrm{ij}} & \tilde{\mathbf{a}}_{\mathrm{ij}}^{\mathrm{T}} \mathbf{P} \tilde{\mathbf{a}}_{\mathrm{ij}}
\end{array}\right]\left[\begin{array}{c}
x(k) \\
w(k) \\
1
\end{array}\right]
\end{aligned}
$$

Converting (19) to an LMI by applying the $S$-procedure $[1,2,10]$, one has

$$
L(x, w, k) \leq \sum_{\mathrm{i}=1}^{\mathrm{r}} \sum_{\mathrm{j}=1}^{\mathrm{r}} h_{\mathrm{i}}(x(k)) h_{\mathrm{j}}(x(k))\left[\begin{array}{c}
x(k) \\
w(k) \\
1
\end{array}\right]^{\mathrm{T}}
$$

$$
\times\left[\begin{array}{ccc}
\boldsymbol{\Phi}_{11} & \boldsymbol{\Phi}_{21}^{\mathrm{T}} & \mathbf{G}_{\mathrm{ij}}^{\mathrm{T}} \mathbf{P} \tilde{\mathbf{a}}_{\mathrm{ij}} \\
\boldsymbol{\Phi}_{21} & \boldsymbol{\Phi}_{22} & \mathbf{N}_{\mathrm{ij}}^{\mathrm{T}} \mathbf{P} \tilde{\mathbf{a}}_{\mathrm{ij}} \\
\tilde{\mathbf{a}}_{\mathrm{ij}}^{\mathrm{T}} \mathbf{P} \mathbf{G}_{\mathrm{ij}} & \tilde{\mathbf{a}}_{\mathrm{ij}}^{\mathrm{T}} \mathbf{P} \mathbf{N}_{\mathrm{ij}} & \tilde{\mathbf{a}}_{\mathrm{ij}}^{\mathrm{T}} \mathbf{P} \tilde{\mathbf{a}}_{\mathrm{ij}}
\end{array}\right]\left[\begin{array}{c}
x(k) \\
w(k) \\
1
\end{array}\right]-\sum_{\mathrm{q}=1}^{\mathrm{p}} \varepsilon_{\mathrm{ijq}} \Theta_{\mathrm{ijq}}(x(k))
$$

where $\Theta_{\mathrm{ijq}}(x(k)) \in \mathfrak{R}$ is defined in (15). Due to $\varepsilon_{\mathrm{ijq}} \geq 0$ and $\Theta_{\mathrm{ijq}}(x(k)) \leq 0$, the $(20)$ can be rewritten as

$$
L(x, w, k) \leq \sum_{\mathrm{i}=1}^{\mathrm{r}} \sum_{\mathrm{j}=1}^{\mathrm{r}} h_{\mathrm{i}}(x(k)) h_{\mathrm{j}}(x(k))\left[\begin{array}{c}
x(k) \\
w(k) \\
1
\end{array}\right]^{\mathrm{T}}
$$

$$
\times\left[\begin{array}{ccc}
\boldsymbol{\Phi}_{11}-\sum_{\mathrm{q}=1}^{\mathrm{p}} \varepsilon_{\mathrm{ijq}} \mathbf{T}_{\mathrm{ijq}} & \boldsymbol{\Phi}_{21}^{\mathrm{T}} & \mathbf{G}_{\mathrm{ij}}^{\mathrm{T}} \mathbf{P} \tilde{\mathbf{a}}_{\mathrm{ij}}-\sum_{\mathrm{q}=1}^{\mathrm{p}} \varepsilon_{\mathrm{ijq}} \mathbf{n}_{\mathrm{ijq}} \\
\boldsymbol{\Phi}_{21} & \mathbf{\Phi}_{22} & \mathbf{N}_{\mathrm{ij}}^{\mathrm{T}} \mathbf{P} \tilde{\mathbf{a}}_{\mathrm{ij}} \\
\tilde{\mathbf{a}}_{\mathrm{ij}}^{\mathrm{T}} \mathbf{P} \mathbf{G}_{\mathrm{ij}}-\sum_{\mathrm{q}=1}^{\mathrm{p}} \varepsilon_{\mathrm{ijq}} \mathbf{n}_{\mathrm{ijq}}^{\mathrm{T}} & \tilde{\mathbf{a}}_{\mathrm{ij}}^{\mathrm{T}} \mathbf{P} \mathbf{N}_{\mathrm{ij}} & \tilde{\mathbf{a}}_{\mathrm{ij}}^{\mathrm{T}} \mathbf{P} \tilde{\mathbf{a}}_{\mathrm{ij}}-\sum_{\mathrm{q}=1}^{\mathrm{p}} \varepsilon_{\mathrm{ijq}} v_{\mathrm{ijq}}
\end{array}\right]\left[\begin{array}{c}
x(k) \\
w(k) \\
1
\end{array}\right]<0
$$

Obviously, if inequality (11) is held for all $x(k) \in \mathrm{X}_{\mathrm{i}}$ and $\mathrm{i} \notin$ $\hat{I}$ then $\sum_{k=0}^{k_{\mathrm{q}}} L(x, w, k)<0$. In this case, one has

$$
J_{D}<0
$$

or

$$
2 \sum_{k=0}^{k_{\mathrm{q}}} y^{\mathrm{T}}(k) w(k)>\sum_{k=0}^{k_{\mathrm{q}}} \gamma w^{\mathrm{T}}(k) w(k)
$$

Since (23) is equivalent to (9), it is easy to show that the system is strictly input passive with exogenous input and output.

Now, we have to show that the discrete-time affine T-S fuzzy model (8) is Lyapunov stable. From (21), if the inequality (11) is held, thus we have $L(x, w, k)<0$. By assuming the exogenous input $w(k)=0$, one has

$$
\Delta V(x(k))<0
$$

Thus, the discrete-time affine T-S fuzzy model (3) is Lyapunov stable and strictly input passive driven by the fuzzy controller (7). Besides, the proof of condition (10) of rules $i \in$ $\hat{I}$ is similar to (11) with setting $\tilde{\mathbf{a}}_{\mathrm{i}}=0$ and assuming $\Theta_{\mathrm{ijq}}(x(k))$ be zero. The proof is completed.

How to compute the $\mathbf{T}_{\mathrm{ijq}}, \mathbf{n}_{\mathrm{ijq}}$ and $v_{\mathrm{ijq}}$ for the $S$-procedure is explained in [10]. In order to achieve the sufficient conditions of (10) and (11), one must find suitable positive definite common matrix $\mathbf{P}=\mathbf{P}^{\mathrm{T}}>0$, scalars $\varepsilon_{\mathrm{ijq}} \geq 0$ and $\gamma \geq 0$. The 
forms of the conditions (10) and (11) are the BMI forms which cannot be calculated by LMI approach for obtaining the solution to satisfy the conditions directly. Hence, the ILMI algorithm need to be developed to solve the problem of BMI and to find the feasible solution for the conditions (10) and (11). By applying some transform techniques, the BMI conditions can be transformed into LMI conditions via the application of auxiliary variables. One of the important transform techniques is Schur complement [1], which is presented in the following lemma.

Lemma 1: (Schur complement) [1]

Nonlinear (convex) matrix inequalities can be converted to LMIs form using Schur complement. Considering the following matrix inequality

$$
\left[\begin{array}{cc}
\mathbf{B}(x) & \mathbf{S}(x) \\
\mathbf{S}^{\mathrm{T}}(x) & \mathbf{T}(x)
\end{array}\right]>0
$$

where $\mathbf{B}(x)=\mathbf{B}^{\mathrm{T}}(x), \mathbf{T}(x)=\mathbf{T}^{\mathrm{T}}(x)$, the (25) can be represented as

$$
\mathbf{T}(x)>0
$$

and

$$
\mathbf{B}(x)-\mathbf{S}(x) \mathbf{T}^{-1}(x) \mathbf{S}^{\mathrm{T}}(x)>0
$$

By applying the auxiliary variables and Schur complement defined in Lemma 1, some new stability conditions can be modified from the conditions (10) and (11) in the next section. Based on these new stability conditions, the feasible solutions of fuzzy controllers can be solved by using the ILMI algorithm.

\section{FUZZY CONTROLLER DESIGN BY USING ILMI ALGORITHM WITH INPUT CONSTRAINTS}

In this section, an ILMI algorithm is applied to obtain fuzzy controller to achieve the conditions of Theorem 1 for discrete-time affine T-S fuzzy model (3). In order to solve the BMI conditions of Theorem 1, we first hold some auxiliary variables as given matrices and then converting it to a LMI problem. After that, the fuzzy controller design problem can be solved by LMI technique. The modified stability conditions are derived in the following theorem.

\section{Theorem 2:}

If there exist positive definite matrices, and scalars, $\varepsilon_{\mathrm{ijq}} \geq 0$ for satisfying the following conditions, then the discrete-time affine T-S fuzzy model (3) is strictly input passive and Lyapunov stable with fuzzy controller (7).

$$
\left\{\begin{array}{l}
\boldsymbol{\Omega}_{\mathrm{ij}}<0 \\
\mathbf{X}^{\mathrm{T}} \mathbf{P X}-\mathbf{X} \leq 0
\end{array} \quad \mathrm{i}, \mathrm{j} \in \hat{I}\right.
$$

and

$$
\left\{\begin{array}{l}
\hat{\boldsymbol{\Omega}}_{\mathrm{ij}}<0 \\
\mathbf{X}^{\mathrm{T}} \mathbf{P X}-\mathbf{X} \leq 0
\end{array} \quad \mathrm{i}, \mathrm{j} \notin \hat{I}\right.
$$

where

$\boldsymbol{\Omega}_{\mathrm{ij}}=\left[\begin{array}{ccc}-\alpha \mathbf{P} & \mathbf{G}_{\mathrm{ij}}^{\mathrm{T}} & \mathbf{G}_{\mathrm{ij}}^{\mathrm{T}} \mathbf{X}^{-1} \mathbf{N}_{\mathrm{ij}}-\mathbf{C}_{\mathrm{i}}^{\mathrm{T}} \\ \mathbf{G}_{\mathrm{ij}} & -\mathbf{X} & 0 \\ \mathbf{N}_{\mathrm{ij}}^{\mathrm{T}} \mathbf{X}^{-1} \mathbf{G}_{\mathrm{ij}}-\mathbf{C}_{\mathrm{i}} & 0 & \mathbf{N}_{\mathrm{ij}}^{\mathrm{T}} \mathbf{X}^{-1} \mathbf{N}_{\mathrm{ij}}-\mathbf{D}_{\mathrm{i}}-\mathbf{D}_{\mathrm{i}}^{\mathrm{T}}+\gamma \mathbf{I}\end{array}\right]$,

$\hat{\mathbf{\Omega}}_{\mathrm{ij}}=\left[\begin{array}{ccc}-\alpha \mathbf{P}-\sum_{\mathrm{q}=1}^{\mathrm{p}} \varepsilon_{\mathrm{ijq}} \mathbf{T}_{\mathrm{ijq}} & \mathbf{G}_{\mathrm{ij}}^{\mathrm{T}} & \mathbf{G}_{\mathrm{ij}}^{\mathrm{T}} \mathbf{X}^{-1} \mathbf{N}_{\mathrm{ij}}-\mathbf{C}_{\mathrm{i}}^{\mathrm{T}} \\ \mathbf{G}_{\mathrm{ij}} & -\mathbf{X} & 0 \\ \mathbf{N}_{\mathrm{ij}}^{\mathrm{T}} \mathbf{X}^{-1} \mathbf{G}_{\mathrm{ij}}-\mathbf{C}_{\mathrm{i}} & 0 & \mathbf{N}_{\mathrm{ij}}^{\mathrm{T}} \mathbf{X}^{-1} \mathbf{N}_{\mathrm{ij}}-\mathbf{D}_{\mathrm{i}}-\mathbf{D}_{\mathrm{i}}^{\mathrm{T}}+\gamma \\ \tilde{\mathbf{a}}_{\mathrm{ij}}^{\mathrm{T}} \mathbf{X}^{-1} \mathbf{G}_{\mathrm{ij}}-\sum_{\mathrm{q}=1}^{\mathrm{p}} \varepsilon_{\mathrm{ijg}} \mathbf{n}_{\mathrm{ijq}}^{\mathrm{T}} & 0 & \tilde{\mathbf{a}}_{\mathrm{ij}}^{\mathrm{T}} \mathbf{X}^{-1} \mathbf{N}_{\mathrm{ij}}\end{array}\right.$

$$
\left.\begin{array}{c}
\mathbf{G}_{\mathrm{ij}}^{\mathrm{T}} \mathbf{X}^{-1} \tilde{\mathbf{a}}_{\mathrm{ij}}-\sum_{\mathrm{q}=1}^{\mathrm{p}} \varepsilon_{\mathrm{ijq}} \mathbf{n}_{\mathrm{ijq}} \\
0 \\
\mathbf{N}_{\mathrm{ij}}^{\mathrm{T}} \mathbf{X}^{-1} \tilde{\mathbf{a}}_{\mathrm{ij}} \\
\tilde{\mathbf{a}}_{\mathrm{ij}}^{\mathrm{T}} \mathbf{X}^{-1} \tilde{\mathbf{a}}_{\mathrm{ij}}-\sum_{\mathrm{q}=1}^{\mathrm{p}} \varepsilon_{\mathrm{ijq}} \mathbf{V}_{\mathrm{ijq}}
\end{array}\right]
$$

\section{Proof:}

Using the Schur complement of Lemma 1, the first condition of (29), i.e., $\hat{\mathbf{\Omega}}_{\mathrm{ij}}<0$, can be rewritten as

$$
\begin{gathered}
{\left[\begin{array}{ccc}
-\alpha \mathbf{P}-\sum_{\mathrm{q}=1}^{\mathrm{p}} \varepsilon_{\mathrm{ijq}} \mathbf{T}_{\mathrm{ijq}} & \mathbf{G}_{\mathrm{ij}}^{\mathrm{T}} & \mathbf{G}_{\mathrm{ij}}^{\mathrm{T}} \mathbf{X}^{-1} \mathbf{N}_{\mathrm{ij}}-\mathbf{C}_{\mathrm{i}}^{\mathrm{T}} \\
\mathbf{G}_{\mathrm{ij}} & -\mathbf{X} & 0 \\
\mathbf{N}_{\mathrm{ij}}^{\mathrm{T}} \mathbf{X}^{-1} \mathbf{G}_{\mathrm{ij}}-\mathbf{C}_{\mathrm{i}} & 0 & \mathbf{N}_{\mathrm{ij}}^{\mathrm{T}} \mathbf{X}^{-1} \mathbf{N}_{\mathrm{ij}}-\mathbf{D}_{\mathrm{i}}-\mathbf{D}_{\mathrm{i}}^{\mathrm{T}}+\gamma \\
\tilde{\mathbf{a}}_{\mathrm{ij}}^{\mathrm{T}} \mathbf{X}^{-1} \mathbf{G}_{\mathrm{ij}}-\sum_{\mathrm{q}=1}^{\mathrm{p}} \varepsilon_{\mathrm{ijq}} \mathbf{n}_{\mathrm{ijq}}^{\mathrm{T}} & 0 & \tilde{\mathbf{a}}_{\mathrm{ij}}^{\mathrm{T}} \mathbf{X}^{-1} \mathbf{N}_{\mathrm{ij}} \\
\mathbf{G}_{\mathrm{ij}}^{\mathrm{T}} \mathbf{X}^{-1} \tilde{\mathbf{a}}_{\mathrm{ij}}-\sum_{\mathrm{q}=1}^{\mathrm{p}} \varepsilon_{\mathrm{ijq}} \mathbf{n}_{\mathrm{ijq}} \\
0 \\
\mathbf{N}_{\mathrm{ij}}^{\mathrm{T}} \mathbf{X}^{-1} \tilde{\mathbf{a}}_{\mathrm{ij}} \\
\tilde{\mathbf{a}}_{\mathrm{ij}}^{\mathrm{T}} \mathbf{X}^{-1} \tilde{\mathbf{a}}_{\mathrm{ij}}-\sum_{\mathrm{q}=1}^{\mathrm{p}} \varepsilon_{\mathrm{ijq}} \mathbf{v}_{\mathrm{ijq}}
\end{array}\right]<\left[\begin{array}{ccc}
(\alpha-1) \mathbf{P} & 0 & 0 \\
0 & 0 & 0 \\
0 & 0 & 0
\end{array}\right]} \\
\end{gathered}
$$


Obviously, if the decay rate and $\mathbf{P}>0$ are held then inequality (30) is strictly negative definite. Multiplying this inequality from left and right by $\left[\begin{array}{lll}x^{\mathrm{T}}(k) & w^{\mathrm{T}}(k) & 1\end{array}\right]$ and, respectively, one can rearrange it as

$$
\begin{aligned}
& x^{\mathrm{T}}(k) \mathbf{G}_{\mathrm{ij}}^{\mathrm{T}} \mathbf{X}^{-1} \mathbf{G} x(k)+x^{\mathrm{T}}(k) \mathbf{G}_{\mathrm{ij}}^{\mathrm{T}} \mathbf{X}^{-1} \mathbf{N}_{\mathrm{ij}} w(k)-x^{\mathrm{T}}(k) \mathbf{C}_{\mathrm{i}}^{\mathrm{T}} w(k) \\
& +x^{\mathrm{T}}(k) \mathbf{G}_{\mathrm{ij}}^{\mathrm{T}} \mathbf{X}^{-1} \tilde{\mathbf{a}}_{\mathrm{ij}}+w^{\mathrm{T}}(k) \mathbf{N}_{\mathrm{ij}}^{\mathrm{T}} \mathbf{X}^{-1} \mathbf{G} x(k)-w^{\mathrm{T}}(k) \mathbf{C}_{\mathrm{i}} x(k) \\
& +w^{\mathrm{T}}(k) \mathbf{N}_{\mathrm{ij}}^{\mathrm{T}} \mathbf{X}^{-1} \mathbf{N}_{\mathrm{ij}} w(k)+\gamma w^{\mathrm{T}}(k) w(k)-w^{\mathrm{T}}(k) \mathbf{D}_{\mathrm{i}}^{\mathrm{T}} w(k) \\
& -w^{\mathrm{T}}(k) \mathbf{D} w(k)+w^{\mathrm{T}}(k) \mathbf{N}_{\mathrm{ij}}^{\mathrm{T}} \mathbf{X}^{-1} \tilde{\mathbf{a}}_{\mathrm{ij}}+\tilde{\mathbf{a}}_{\mathrm{ij}}^{\mathrm{T}} \mathbf{X}^{-1} \mathbf{G} x(k)+\tilde{\mathbf{a}}_{\mathrm{ij}}^{\mathrm{T}} \mathbf{X}^{-1} \\
& \times \mathbf{N}_{\mathrm{ij}} w(k)+\tilde{\mathbf{a}}_{\mathrm{ij}}^{\mathrm{T}} \mathbf{X}^{-1} \tilde{\mathbf{a}}_{\mathrm{ij}}-x^{\mathrm{T}}(k) \mathbf{P} x(k)-\sum_{\mathrm{q}=1}^{\mathrm{p}} \varepsilon_{\mathrm{ijq}} \Theta_{\mathrm{ijq}}(x(k)) \\
& <(\alpha-1) x^{\mathrm{T}}(k) \mathbf{P} x(k)
\end{aligned}
$$

or

$$
\begin{aligned}
\left(\mathbf{G}_{\mathrm{ij}} x(k)\right. & \left.+\mathbf{N}_{\mathrm{ij}} w(k)+\tilde{\mathbf{a}}_{\mathrm{ij}}\right)^{\mathrm{T}} \mathbf{X}^{-1}\left(\mathbf{G}_{\mathrm{ij}} x(k)+\mathbf{N}_{\mathrm{ij}} w(k)+\tilde{\mathbf{a}}_{\mathrm{ij}}\right) \\
& -x^{\mathrm{T}}(k) \mathbf{P} x(k)+\gamma w^{\mathrm{T}}(k) w(k)-2 y^{\mathrm{T}}(k) w(k) \\
& <(\alpha-1) x^{\mathrm{T}}(k) \mathbf{P} x(k)
\end{aligned}
$$

If there exist $\mathbf{P}>0$ such that $\mathbf{X}^{\mathrm{T}} \mathbf{P X}-\mathbf{X} \leq 0$ is held, one can obtain the following inequality according to (32).

$$
\begin{aligned}
\left(\mathbf{G}_{\mathrm{ij}} x(k)\right. & \left.+\mathbf{N}_{\mathrm{ij}} w(k)+\tilde{\mathbf{a}}_{\mathrm{ij}}\right)^{\mathrm{T}} \mathbf{P}\left(\mathbf{G}_{\mathrm{ij}} x(k)+\mathbf{N}_{\mathrm{ij}} w(k)+\tilde{\mathbf{a}}_{\mathrm{ij}}\right) \\
& -x^{\mathrm{T}}(k) \mathbf{P} x(k)+\gamma w^{\mathrm{T}}(k) w(k)-2 y^{\mathrm{T}}(k) w(k) \\
& -\sum_{\mathrm{q}=1}^{\mathrm{p}} \varepsilon_{\mathrm{ijq}} \Theta_{\mathrm{ijq}}(x(k))<(\alpha-1) x^{\mathrm{T}}(k) \mathbf{P} x(k)
\end{aligned}
$$

or

$$
\begin{aligned}
{\left[\begin{array}{c}
x(k) \\
w(k) \\
1
\end{array}\right]\left[\begin{array}{ccc}
\mathbf{\Phi}_{11}-\sum_{\mathrm{q}=1}^{\mathrm{p}} \varepsilon_{\mathrm{ijq}} \mathbf{T}_{\mathrm{ijq}} & \mathbf{\Phi}_{21}^{\mathrm{T}} & \mathbf{G}_{\mathrm{ij}}^{\mathrm{T}} \mathbf{P} \tilde{\mathbf{a}}_{\mathrm{ij}}-\sum_{\mathrm{q}=1}^{\mathrm{p}} \varepsilon_{\mathrm{ijq}} \mathbf{n}_{\mathrm{ijq}} \\
\mathbf{\Phi}_{21} & \mathbf{\Phi}_{22} & \mathbf{N}_{\mathrm{ij}}^{\mathrm{T}} \mathbf{P} \tilde{\mathbf{a}}_{\mathrm{ij}} \\
\tilde{\mathbf{a}}_{\mathrm{ij}}^{\mathrm{T}} \mathbf{P} \mathbf{G}_{\mathrm{ij}}-\sum_{\mathrm{q}=1}^{\mathrm{p}} \varepsilon_{\mathrm{ijq}} \mathbf{n}_{\mathrm{ijq}}^{\mathrm{T}} & \tilde{\mathbf{a}}_{\mathrm{ij}}^{\mathrm{T}} \mathbf{P} \mathbf{N}_{\mathrm{ij}} & \tilde{\mathbf{a}}_{\mathrm{ij}}^{\mathrm{T}} \mathbf{P} \tilde{\mathbf{a}}_{\mathrm{ij}}-\sum_{\mathrm{q}=1}^{\mathrm{p}} \varepsilon_{\mathrm{ijq}} v_{\mathrm{ijq}}
\end{array}\right] } \\
\times\left[\begin{array}{c}
x(k) \\
w(k) \\
1
\end{array}\right]<\left[\begin{array}{c}
x(k) \\
w(k) \\
1
\end{array}\right]^{\mathrm{T}}\left[\begin{array}{ccc}
(\alpha-1) \mathbf{P} & 0 & 0 \\
0 & 0 & 0 \\
0 & 0 & 0
\end{array}\right]\left[\begin{array}{c}
x(k) \\
w(k) \\
1
\end{array}\right]
\end{aligned}
$$

Obviously,

$$
\begin{aligned}
& {\left[\begin{array}{ccc}
\boldsymbol{\Phi}_{11}-\sum_{\mathrm{q}=1}^{\mathrm{p}} \varepsilon_{\mathrm{ijq}} \mathbf{T}_{\mathrm{ijq}} & \boldsymbol{\Phi}_{21}^{\mathrm{T}} & \mathbf{G}_{\mathrm{ij}}^{\mathrm{T}} \mathbf{P} \tilde{\mathbf{a}}_{\mathrm{ij}}-\sum_{\mathrm{q}=1}^{\mathrm{p}} \varepsilon_{\mathrm{ijq}} \mathbf{n}_{\mathrm{ijq}} \\
\boldsymbol{\Phi}_{21} & \mathbf{\Phi}_{22} & \mathbf{N}_{\mathrm{ij}}^{\mathrm{T}} \mathbf{P} \tilde{\mathbf{a}}_{\mathrm{ij}} \\
\tilde{\mathbf{a}}_{\mathrm{ij}}^{\mathrm{T}} \mathbf{P} \mathbf{G}_{\mathrm{ij}}-\sum_{\mathrm{q}=1}^{\mathrm{p}} \varepsilon_{\mathrm{ijq}} \mathbf{n}_{\mathrm{ijq}}^{\mathrm{T}} & \tilde{\mathbf{a}}_{\mathrm{ij}}^{\mathrm{T}} \mathbf{P} \mathbf{N}_{\mathrm{ij}} & \tilde{\mathbf{a}}_{\mathrm{ij}}^{\mathrm{T}} \mathbf{P} \tilde{\mathbf{a}}_{\mathrm{ij}}-\sum_{\mathrm{q}=1}^{\mathrm{p}} \varepsilon_{\mathrm{ijq}} \nu_{\mathrm{ijq}}
\end{array}\right]} \\
& <\left[\begin{array}{ccc}
(\alpha-1) \mathbf{P} & 0 & 0 \\
0 & 0 & 0 \\
0 & 0 & 0
\end{array}\right]
\end{aligned}
$$

Since $\mathbf{P}>0$ and $\alpha<1$, one has

$$
\left[\begin{array}{ccc}
\boldsymbol{\Phi}_{11}-\sum_{\mathrm{q}=1}^{\mathrm{p}} \varepsilon_{\mathrm{ijq}} \mathbf{T}_{\mathrm{ijq}} & \boldsymbol{\Phi}_{21}^{\mathrm{T}} & \mathbf{G}_{\mathrm{ij}}^{\mathrm{T}} \mathbf{P} \tilde{\mathbf{a}}_{\mathrm{ij}}-\sum_{\mathrm{q}=1}^{\mathrm{p}} \varepsilon_{\mathrm{ijq}} \mathbf{n}_{\mathrm{ijq}} \\
\boldsymbol{\Phi}_{21} & \boldsymbol{\Phi}_{22} & \mathbf{N}_{\mathrm{ij}}^{\mathrm{T}} \mathbf{P} \tilde{\mathbf{a}}_{\mathrm{ij}} \\
\tilde{\mathbf{a}}_{\mathrm{ij}}^{\mathrm{T}} \mathbf{P} \mathbf{G}_{\mathrm{ij}}-\sum_{\mathrm{q}=1}^{\mathrm{p}} \varepsilon_{\mathrm{ijq}} \mathbf{n}_{\mathrm{ijq}}^{\mathrm{T}} & \tilde{\mathbf{a}}_{\mathrm{ij}}^{\mathrm{T}} \mathbf{P} \mathbf{N}_{\mathrm{ij}} & \tilde{\mathbf{a}}_{\mathrm{ij}}^{\mathrm{T}} \mathbf{P} \tilde{\mathbf{a}}_{\mathrm{ij}}-\sum_{\mathrm{q}=1}^{\mathrm{p}} \varepsilon_{\mathrm{ijq}} v_{\mathrm{ijq}}
\end{array}\right]<0
$$

The inequality (36) is equivalent to (11). Hence, in the case of $\mathrm{i} \notin \hat{I}$, if condition (29) is satisfied then the discrete-time affine T-S fuzzy model is strictly input passive and Lyapunov stable. The proof of (28) for rule including the origin $(\mathrm{i} \in \hat{I})$ is similar to (29) by setting $\tilde{\mathbf{a}}_{\mathrm{i}}$ and $\Theta_{\mathrm{ijq}}$ be all zero. Hence, the proof of (28) is omitted here and the proof of this theorem is completed.

The conditions of Theorem 2 provide the stability conditions for achieving the passivity property of discrete affine T-S fuzzy models. However, the control input constraint was not considered in these conditions. For achieving the input constraint, the following theorem provide the LMI conditions such that

\section{Theorem 3:}

Considering the system and conditions described in Theorem 2, assume that the initial condition $x(0)$ is known. The input constraint $\|u(k)\|_{2} \leq \mu$ is enforced at all times $k \geq 0$ if the following LMI conditions are satisfied.

$$
\begin{gathered}
{\left[\begin{array}{cc}
1 & x(0)^{\mathrm{T}} \\
x(0) & \mathbf{X}
\end{array}\right] \geq 0} \\
{\left[\begin{array}{cc}
\mathbf{X} & \mathbf{M}_{\mathrm{i}}^{\mathrm{T}} \\
\mathbf{M}_{\mathrm{i}} & \mu^{2} \mathbf{I}
\end{array}\right] \geq 0}
\end{gathered}
$$

where $\mathbf{X}=\mathbf{P}^{-1}, \mathbf{M}_{\mathrm{i}}=\mathbf{F}_{\mathrm{i}} \mathbf{X}$.

\section{Proof:}

Assume that $V(x(k))=x^{\mathrm{T}}(k) \mathbf{P} x(k)$ is a Lyapunov function and $x^{\mathrm{T}}(0) \mathbf{P} x(0) \leq 1$. Then, 


$$
1-x^{\mathrm{T}}(0) \mathbf{P} x(0) \geq 0
$$

where $\mathbf{X}=\mathbf{P}^{-1}$. The inequality (39) can be transformed into (37) by the Schur complement procedure.

The derivation of (38) is stated as follows. From $\|u(k)\|_{2} \leq$ $\mu$, one has

$$
u^{\mathrm{T}}(k) u(k)=\sum_{\mathrm{i}=1}^{\mathrm{r}} \sum_{\mathrm{j}=1}^{\mathrm{r}} h_{\mathrm{i}}(x(k)) h_{\mathrm{j}}(x(k)) x^{\mathrm{T}}(k) \mathbf{F}_{\mathrm{i}}^{\mathrm{T}} \mathbf{F}_{\mathrm{j}} x(k) \leq \mu^{2}
$$

Therefore,

$$
\frac{1}{\mu^{2}} \sum_{\mathrm{i}=1}^{\mathrm{r}} \sum_{\mathrm{j}=1}^{\mathrm{r}} h_{\mathrm{i}}(x(k)) h_{\mathrm{j}}(x(k)) x^{\mathrm{T}}(k) \mathbf{F}_{\mathrm{i}}^{\mathrm{T}} \mathbf{F}_{\mathrm{j}} x(k) \leq 1
$$

Since $x^{\mathrm{T}}(k) \mathbf{X}^{-1} x(k)<x^{\mathrm{T}}(0) \mathbf{X}^{-1} x(0) \leq 1$ for $k>0$, if

$\frac{1}{\mu^{2}} \sum_{\mathrm{i}=1}^{\mathrm{r}} \sum_{\mathrm{j}=1}^{\mathrm{r}} h_{\mathrm{i}}(x(k)) h_{\mathrm{j}}(x(k)) x^{\mathrm{T}}(k) \mathbf{F}_{\mathrm{i}}^{\mathrm{T}} \mathbf{F}_{\mathrm{j}} x(k) \leq x^{\mathrm{T}}(k) \mathbf{X}^{-1} x(k)$

then (41) holds. Therefore, one has

$$
\sum_{\mathrm{i}=1}^{\mathrm{r}} \sum_{\mathrm{j}=1}^{\mathrm{r}} h_{\mathrm{i}}(x(k)) h_{\mathrm{j}}(x(k)) x^{\mathrm{T}}(k)\left(\frac{1}{\mu^{2}} \mathbf{F}_{\mathrm{i}}^{T} \mathbf{F}_{\mathrm{j}}-\mathbf{X}^{-1}\right) x(k) \leq 0
$$

From the left-hand side of (42), one has

$$
\begin{aligned}
\frac{1}{2} \sum_{\mathrm{i}=1}^{\mathrm{r}} \sum_{\mathrm{j}=1}^{\mathrm{r}} h_{\mathrm{i}}(x(k)) h_{\mathrm{j}}(x(k)) x^{\mathrm{T}}(k)\left(\frac{1}{\mu^{2}} \mathbf{F}_{\mathrm{i}}^{\mathrm{T}} \mathbf{F}_{\mathrm{j}}+\frac{1}{\mu^{2}} \mathbf{F}_{\mathrm{j}}^{\mathrm{T}} \mathbf{F}_{\mathrm{i}}-2 \mathbf{X}^{-1}\right) x(k) \\
=\frac{1}{2} \sum_{\mathrm{i}=1}^{\mathrm{r}} \sum_{\mathrm{j}=1}^{\mathrm{r}} h_{\mathrm{i}}(x(k)) h_{\mathrm{j}}(x(k)) x^{\mathrm{T}}(k)\left(\frac{1}{\mu^{2}}\left(\mathbf{F}_{\mathrm{i}}^{\mathrm{T}} \mathbf{F}_{\mathrm{j}}+\mathbf{F}_{\mathrm{j}}^{\mathrm{T}} \mathbf{F}_{\mathrm{i}}\right)\right. \\
\left.\quad-\frac{1}{\mu^{2}}\left(\mathbf{F}_{\mathrm{i}}^{\mathrm{T}}-\mathbf{F}_{\mathrm{j}}^{\mathrm{T}}\right)\left(\mathbf{F}_{\mathrm{i}}-\mathbf{F}_{\mathrm{j}}\right)-2 \mathbf{X}^{-1}\right) x(k) \\
\leq \sum_{\mathrm{i}=1}^{\mathrm{r}} \sum_{\mathrm{j}=1}^{\mathrm{r}} h_{\mathrm{i}}(x(k)) h_{\mathrm{j}}(x(k)) x^{\mathrm{T}}(k)\left(\frac{1}{\mu^{2}}\left(\mathbf{F}_{\mathrm{i}}^{\mathrm{T}} \mathbf{F}_{\mathrm{j}}+\mathbf{F}_{\mathrm{j}}^{\mathrm{T}} \mathbf{F}_{\mathrm{i}}\right)-2 \mathbf{X}^{-1}\right) \\
\quad \times x(k) \\
=\sum_{\mathrm{i}=1}^{\mathrm{r}} h_{\mathrm{i}}(x(k)) x^{\mathrm{T}}(k)\left(\frac{1}{\mu^{2}} \mathbf{F}_{\mathrm{i}}^{\mathrm{T}} \mathbf{F}_{\mathrm{i}}-\mathbf{X}^{-1}\right) x(k)
\end{aligned}
$$

Thus, if

$$
\frac{1}{\mu^{2}} \mathbf{F}_{\mathrm{i}}^{\mathrm{T}} \mathbf{F}_{\mathrm{i}}-\mathbf{X}^{-1} \leq 0
$$

then (42) holds. By defining $\mathbf{M}_{\mathrm{i}}=\mathbf{F}_{\mathrm{i}} \mathbf{X}$, one can obtain

$$
\frac{1}{\mu^{2}} \mathbf{M}_{\mathrm{i}}^{\mathrm{T}} \mathbf{M}_{\mathrm{i}}-\mathbf{X} \leq 0
$$

Inequality (38) can be obtained from the above inequality by the Schur complement procedure.

In order to achieve the conditions (28), (29), (37) and (38), an ILMI algorithm is developed to interactively search for $\mathbf{P}$, $\mathbf{F}_{\mathrm{i}}, \alpha, \varepsilon_{\mathrm{ijq}}$ and to update the auxiliary variable $\mathbf{X}$ until $\alpha<1$. The operation process of the present ILMI algorithm is proposed as follows.

\section{ILMI Algorithm}

Step 1: Define the iterative auxiliary variable as follows.

$$
\mathbf{X}_{(\mathrm{t})}=\mathbf{P}_{(\mathrm{t}-1)}^{-1}
$$

where $t$ is iterative index. Set $t=1$ and the initial $\mathbf{P}_{(0)}$ can be calculated by the following equation.

$$
\hat{\mathbf{A}}^{\mathrm{T}} \mathbf{P}_{(0)} \hat{\mathbf{A}}-\mathbf{P}_{(0)}-\left(\hat{\mathbf{A}}^{\mathrm{T}} \mathbf{P}_{(0)} \hat{\mathbf{B}}\right)\left(1+\hat{\mathbf{B}}^{\mathrm{T}} \mathbf{P}_{(0)} \hat{\mathbf{B}}\right)^{-1}\left(\hat{\mathbf{B}}^{\mathrm{T}} \mathbf{P}_{(0)} \hat{\mathbf{A}}\right)+\mathbf{Q}=\mathbf{0}
$$

where $\hat{\mathbf{A}}=\frac{1}{\mathrm{r}} \sum_{\mathrm{i}=1}^{\mathrm{r}} \mathbf{A}_{\mathrm{i}}, \hat{\mathbf{B}}=\frac{1}{\mathrm{r}} \sum_{\mathrm{i}=1}^{\mathrm{r}} \mathbf{B}_{\mathrm{i}}$, and $\mathbf{Q}>0$. The matrix $\mathbf{Q}$ is assigned by the designers. Then start the ILMI algorithm.

Step 2: Use the auxiliary variable $\mathbf{X}_{(\mathrm{t})}=\mathbf{P}_{(\mathrm{t}-1)}^{-1}$ to solve the optimization problem for $\mathbf{P}_{(\mathrm{t})}, \mathbf{F}_{\mathrm{i}}, \boldsymbol{\varepsilon}_{\mathrm{ijq}(\mathrm{t})}$ from (28) and (29) subject to minimizing $\alpha_{(\mathrm{t})}$. That is,

Minimize $\alpha_{(\mathrm{t})}$

Subject to $\mathbf{P}_{(\mathrm{t})}>0, \varepsilon_{\mathrm{ijg}(\mathrm{t})}>0$, (28) for i, $\mathrm{j} \in \hat{I}$ and (29) for $\mathrm{i}, \mathrm{j} \notin \hat{I}$.

If $\alpha_{(\mathrm{t})}<1$, then $\mathbf{P}_{(\mathrm{t})}, \mathbf{F}_{\mathrm{i}}$ and $\varepsilon_{\mathrm{ijg}(\mathrm{t})}$ obtained in Step 2 are the feasible solutions for the conditions (28) and (29). Then go to Step 4. Otherwise, go to Step 3.

Step 3. Resolve the following optimization problem for $\mathbf{P}_{(\mathrm{t})}$, $\mathbf{F}_{\mathrm{i}}$ and $\varepsilon_{\mathrm{ijg}(\mathrm{t})}$ using $\alpha_{(\mathrm{t})}$ determined in Step 2 and auxiliary variable $\mathbf{X}_{(\mathrm{t})}$ obtained in Step 2.

Minimize trace $\left(\mathbf{P}_{(\mathrm{t})}\right)$

Subject to $\mathbf{P}_{(\mathrm{t})}>0, \varepsilon_{\mathrm{ijg}(\mathrm{t})}>0$, (28) for $\mathrm{i}, \mathrm{j} \in \hat{I}$ and (29) for $\mathrm{i}, \mathrm{j} \notin \hat{I}$.

If $\left\|\mathbf{X}_{(\mathrm{t})}^{-1}-\mathbf{P}_{(\mathrm{t})}\right\|<\tau$, where $\tau$ is the predetermined small value, then the conditions (28) and (29) are not feasible and then stop the iterative manner. Otherwise, set $t=t+1$ and go back to the Step 2 for updating the auxiliary variable $\mathbf{X}_{(\mathrm{t})}$ using $\mathbf{P}_{(\mathrm{t}-1)}^{-1}$, where $\mathbf{P}_{(t-1)}^{-1}$ is determined in Step 3. 
Step 4: Using the matrix $\mathbf{P}$ determined in Step 2 to obtain the auxiliary variable $\mathbf{X}=\mathbf{P}^{-1}$ and then to solve the following optimization problem for $\mathbf{P}, \mathbf{F}_{\mathrm{i}}$ and $\varepsilon_{\mathrm{ijq}}$.

Minimize $\mu$

Subject to $\mathbf{P}>0, \varepsilon_{\mathrm{ijq}}>0, \mathbf{X}^{\mathrm{T}} \mathbf{P X}-\mathbf{X} \leq 0,(28)$ for $\mathrm{i}, \mathrm{j} \in \hat{I},(29)$ for $\mathrm{i}, \mathrm{j} \notin \hat{I}$ and (37)-(38).

The minimized value $\mu$ is the minimum upper bound for the input norm constraint. The parameters obtained in this step are the final feasible solutions and the algorithm is stop in this step.

The input energy constrained fuzzy control problem considered in this paper can be solved by using the above ILMI algorithm. According to the above ILIMI algorithm, a numerical example is presented to show the application of proposed fuzzy controller design approach for discrete-time affine T-S fuzzy model. Using the proposed fuzzy controller design procedure, the feasible solutions $\mathbf{P}>0, \mathbf{F}_{\mathrm{i}}$ and $\varepsilon_{\mathrm{ijq}}>0$ can be solved by the LMI toolbox of MATLAB to satisfy conditions (28), (29), (37) and (38), simultaneously.

\section{APPLICATION TO CONTROL THE NONLINEAR TRUCK-TRAILER SYSTEM}

In this section, a discrete nonlinear truck-trailer system [17] shown in Fig. 1 is considered to test and verify the fuzzy controller design approach developed in Section 3. It is well known that backing up control of truck-trailers is a difficult exercise since its dynamics is nonlinear and unstable. The specific problem in this simulation is to back up a truck-trailer from an arbitrary initial position by manipulating the steering. Referring to the literature [17], the dynamics of discrete nonlinear truck-trailer system can be characterized as follows.

$$
\begin{gathered}
x_{1}(k+1)=\left(1-\frac{v t}{L_{1}}\right) x_{1}(k)+\frac{v t}{L_{2}} u(k)+0.1 w(k), \\
x_{2}(k+1)=\frac{v t}{L_{1}} x_{1}(k)+x_{2}(k), \\
x_{3}(k+1)=x_{3}(k)+v t \sin \left(x_{2}(k)+\left(v t / 2 L_{1}\right) x_{1}(k)\right), \\
y(k)=x_{1}(k)+w(k)
\end{gathered}
$$

where $x_{1}(k)$ is the angle difference between truck and trailer; $x_{2}(k)$ is the angle of trailer; $x_{3}(k)$ is the vertical position of rear end of trailer; $w(k)$ is the exogenous input provided by a random which noise with variance 1 . Note that $x_{1}(k)$ corresponds to two "jackknife" positions, $90^{\circ}$ and $-90^{\circ}$. The sampling

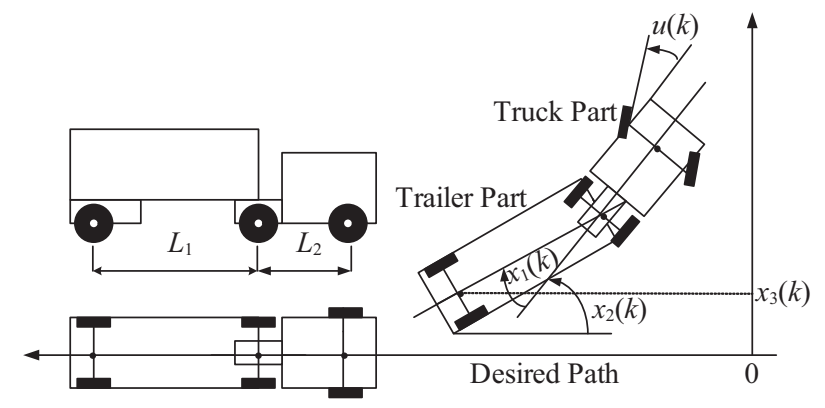

Fig. 1. Nonlinear truck-trailer system.

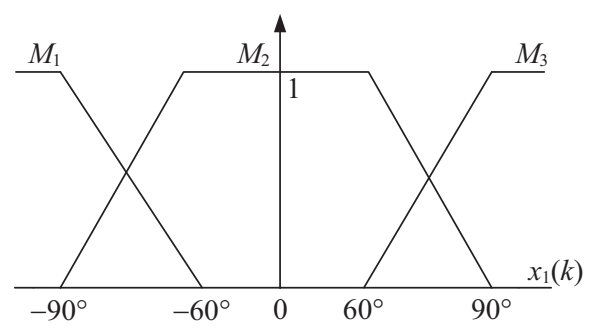

Fig. 2. Membership function of $x_{1}(k)$.

period $t=2 \mathrm{sec}$ and the length of trailer $L_{1}=5.5 \mathrm{~m}$, the length of truck $L_{2}=2.8 \mathrm{~m}$, the constant speed of the backward movement $v=-1 \mathrm{~m} / \mathrm{sec}$. Appling the above system parameters, the dynamic equation (47) can be thus represented as follows.

$$
\begin{gathered}
x_{1}(k+1)=1.3636 x_{1}(k)-0.7143 u(k)+0.1 w(k) \\
x_{2}(k+1)=x_{2}(k)-0.3636 x_{1}(k) \\
x_{3}(k+1)=x_{3}(k)-2 \sin \left(x_{2}(k)-0.1818 x_{1}(k)\right) \\
y(k)=x_{1}(k)+w(k)
\end{gathered}
$$

It can be found that the equilibrium point of (48) is $x_{1}(k)=$ $x_{2}(k)=x_{3}(k)=u(k)=w(k)=0$. In addition to the equilibrium point, we choose other two operating points for obtaining the T-S fuzzy models of the truck-trailer system (48). These operating points are given as follows.

$$
\begin{gathered}
\left(x^{-}, u^{-}, w^{-}\right)_{\text {oper } 1}=\left(\begin{array}{lll|l}
-88^{\circ} & -90^{\circ} & -20|0| 0
\end{array}\right) \\
(x, u, w)_{\text {oper } 2}=\left(\begin{array}{lll|l|l}
0^{\circ} & 0^{\circ} & 0 & 0 \mid 0
\end{array}\right) \\
\left(x^{+}, u^{+}, w^{+}\right)_{\text {oper } 3}=\left(\begin{array}{lllll}
88^{\circ} & 90^{\circ} & 20|0| 0
\end{array}\right)
\end{gathered}
$$

The structure of affine T-S fuzzy model of the nonlinear truck-trailer system (48) can be constructed by linearization method through the above three linear operating points with defining the membership function as Fig. 2. Thus, one can 
represent the discrete affine T-S fuzzy model, which is composed by three fuzzy rules, as follows.

\section{Plant Part:}

Rule 1: IF $x_{1}(k)$ is $M_{1}$ THEN

$$
\begin{gathered}
x(k+1)=\left[\begin{array}{ccc}
1.3636 & 0 & 0 \\
-0.3636 & 1 & 0 \\
0.1002 & -0.5513 & 1
\end{array}\right] x(k)+\left[\begin{array}{c}
-0.7143 \\
0 \\
0
\end{array}\right] u(k) \\
+\left[\begin{array}{c}
0.1 \\
0 \\
0
\end{array}\right] w(k)+\left[\begin{array}{c}
0 \\
0 \\
1.2105
\end{array}\right] \\
y(k)=\left[\begin{array}{lll}
1 & 0 & 0
\end{array}\right] x(k)+w(k)
\end{gathered}
$$

Rule 2: IF $x_{1}(k)$ is $M_{2}$ THEN

$$
\begin{gathered}
x(k+1)=\left[\begin{array}{ccc}
1.3636 & 0 & 0 \\
-0.3636 & 1 & 0 \\
0.3636 & -2 & 1
\end{array}\right] x(k)+\left[\begin{array}{c}
-0.7143 \\
0 \\
0
\end{array}\right] u(k) \\
+\left[\begin{array}{c}
0.1 \\
0 \\
0
\end{array}\right] w(k) \\
y(k)=\left[\begin{array}{lll}
1 & 0 & 0
\end{array}\right] x(k)+w(k)
\end{gathered}
$$

Rule 3: IF $x_{1}(k)$ is $M_{3}$ THEN

$$
\begin{gathered}
x(k+1)=\left[\begin{array}{ccc}
1.3636 & 0 & 0 \\
-0.3636 & 1 & 0 \\
0.1002 & -0.5513 & 1
\end{array}\right] x(k)+\left[\begin{array}{c}
-0.7143 \\
0 \\
0
\end{array}\right] u(k) \\
+\left[\begin{array}{c}
0.1 \\
0 \\
0
\end{array}\right] w(k)+\left[\begin{array}{c}
0 \\
0 \\
-1.2105
\end{array}\right] \\
y(k)=\left[\begin{array}{lll}
1 & 0 & 0
\end{array}\right] x(k)+w(k)
\end{gathered}
$$

The corresponding matrices of $S$-procedure $[1,2,10]$ are presented as follows.

For Rule $11,-90^{\circ} \leq x_{1}(k) \leq-60^{\circ}$ which means

$$
\begin{gathered}
\mathbf{T}_{111}=\left[\begin{array}{lll}
1 & 0 & 0 \\
0 & 0 & 0 \\
0 & 0 & 0
\end{array}\right], \mathbf{n}_{111}=\left[\begin{array}{c}
-((-60-90 \pi / 180) / 2 \\
0 \\
0
\end{array}\right] \text { and } \\
v_{111}=(-60 \pi / 180) \times(-90 \pi / 180)
\end{gathered}
$$

For Rule $31,90^{\circ} \geq x_{1}(k) \geq 60^{\circ}$ which means

$$
\begin{gathered}
\mathbf{T}_{331}=\left[\begin{array}{lll}
1 & 0 & 0 \\
0 & 0 & 0 \\
0 & 0 & 0
\end{array}\right], \mathbf{n}_{331}=\left[\begin{array}{c}
-((60+90) \pi / 180) / 2 \\
0 \\
0
\end{array}\right] \text { and } \\
v_{331}=(60 \pi / 180) \times(90 \pi / 180)
\end{gathered}
$$

where the notion Rule ij means the correlation between Rule and Rule j of the plant part.

For the fuzzy controller design of truck-trailer system (47), the control purpose is to realize the backward movement of the truck-trailer system to guarantee the Lyapunov stability and passivity for the closed-loop system. Through the above discrete affine T-S fuzzy model (50), the fuzzy controller can be designed by applying Theorem 2 and Theorem 3 as well as fuzzy controller design procedure developed in ILMI algorithm. First, let us choose the dissipative rate $\gamma=1$ and the matrix $\mathbf{Q}$ such as

$$
\mathbf{Q}=\left[\begin{array}{lll}
1 & 0 & 0 \\
0 & 1 & 0 \\
0 & 0 & 1
\end{array}\right]
$$

The initial $\mathbf{P}_{(0)}$ can be solved via Riccati Eq. (46) as follows.

$$
\mathbf{P}_{(0)}=\left[\begin{array}{ccc}
99.892 & -62.001 & 11.0722 \\
-62.001 & 121.4182 & -21.4873 \\
11.0722 & -21.4873 & 7.0375
\end{array}\right]
$$

Thus, the matrix $\mathbf{X}_{(1)}$ can be obtained from (45).

$$
\mathbf{X}_{(1)}=\mathbf{P}_{(0)}^{-1}=\left[\begin{array}{ccc}
0.0147 & 0.0074 & -0.0005 \\
0.0074 & 0.0217 & 0.0545 \\
-0.0005 & 0.0545 & 0.3091
\end{array}\right]
$$

Using the above initial auxiliary parameters, one can obtain the minimum $\alpha$ from Step 2 of the proposed ILMI algorithm. If $\alpha<1$, then one can obtain the feasible solution for fuzzy controllers. Otherwise, if $\alpha \geq 1$, a new matrix $\mathbf{P}_{(1)}$ should be re-calculated from the Step 3 and then be substituted into Step 2 to produce new auxiliary parameter $\mathbf{X}_{(2)}$. Repeating this ILMI algorithm until $\alpha<1$, one can obtain the feasible solution of suitable fuzzy controllers. In this example, the feasible solution is obtained after six iterations of the proposed ILMI algorithm. The final $\alpha$ is 0.9832 and the feasible solution is obtained as follows.

$$
\mathbf{P}=\left[\begin{array}{ccc}
13.9962 & -27.5528 & 3.3429 \\
-27.5528 & 96.8441 & -16.6789 \\
3.3429 & -16.6789 & 3.4385
\end{array}\right]
$$




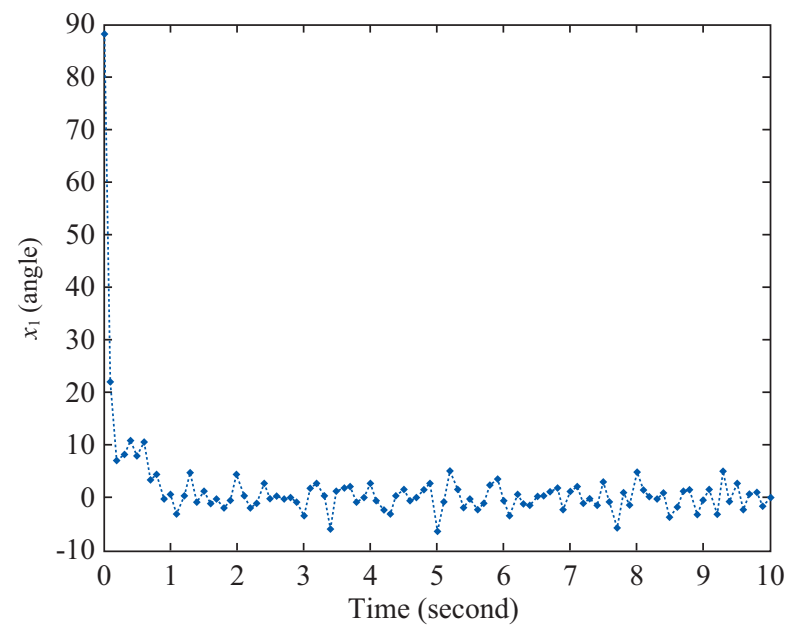

Fig. 3. Responses of state $x_{1}(k)$.

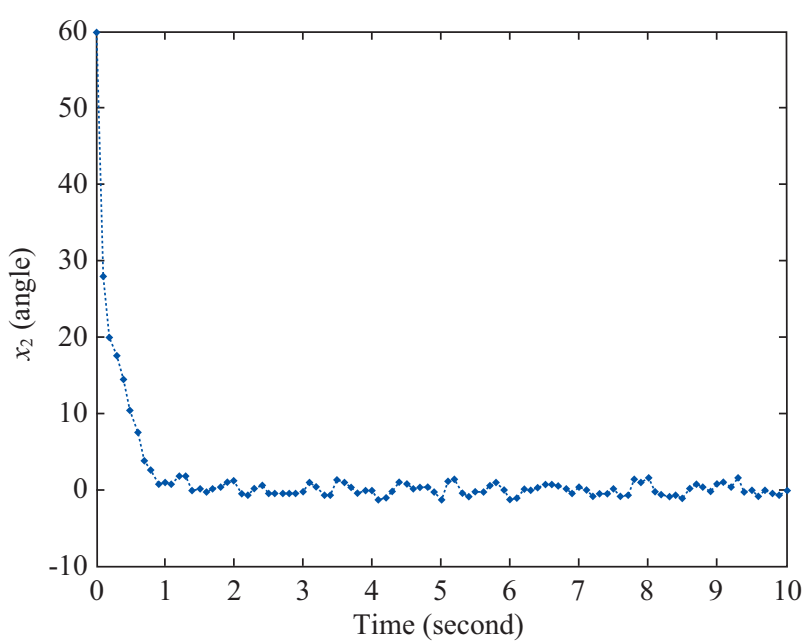

Fig. 4. Responses of state $x_{2}(k)$.

$$
\begin{aligned}
\mathbf{X} & =\left[\begin{array}{lll}
0.3643 & 0.2584 & 0.8963 \\
0.2584 & 0.2439 & 0.9288 \\
0.8963 & 0.9288 & 3.9093
\end{array}\right], \\
\mathbf{F}_{1} & =\left[\begin{array}{lll}
-2.5023 & 3.1145 & -0.3749
\end{array}\right], \\
\mathbf{F}_{2} & =\left[\begin{array}{lll}
-2.2118 & 3.4128 & -0.457
\end{array}\right], \\
\mathbf{F}_{3} & =\left[\begin{array}{lll}
-2.5023 & 3.1145 & -0.3749
\end{array}\right] \\
\mu=2.7103 &
\end{aligned}
$$

and

$$
\varepsilon_{111}=\varepsilon_{331}=19.6752
$$

Based on the above feasible solutions, the PDC-based fuzzy controller can be obtained as follows.

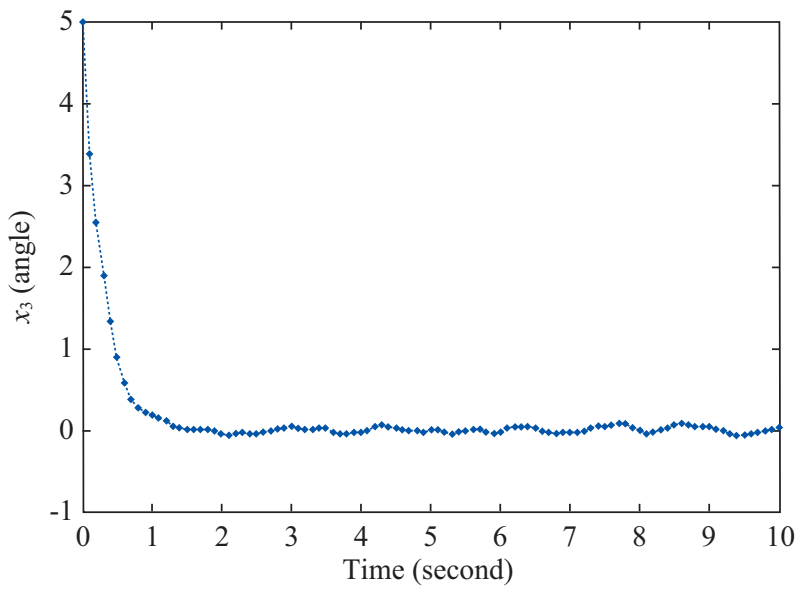

Fig. 5. Responses of state $x_{3}(k)$.

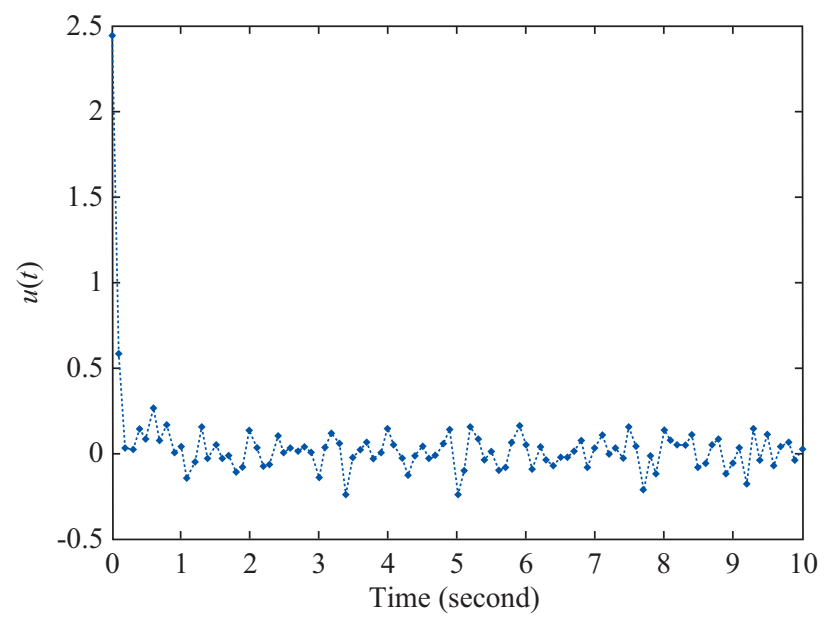

Fig. 6. Responses of control input $u(t)$.

\section{Controller Part:}

Rule 1: IF $x_{1}(k)$ is $M_{1}$ THEN

$$
u(k)=\left[\begin{array}{lll}
-2.5023 & 3.1145 & -0.3749
\end{array}\right] x(k),
$$

Rule 2: IF $x_{1}(k)$ is $M_{2}$ THEN

$$
u(k)=\left[\begin{array}{lll}
-2.2118 & 3.4128 & -0.457
\end{array}\right] x(k),
$$

Rule 3: IF $x_{1}(k)$ is $M_{3}$ THEN

$$
u(k)=\left[\begin{array}{lll}
-2.5023 & 3.1145 & -0.3749
\end{array}\right] x(k),
$$

Setting the initial condition as $x(0)=\left[\begin{array}{lll}88^{0} & 60^{\circ} & 5\end{array}\right]^{\mathrm{T}}$, the simulation results of states and intput are shown in Fig. 3 to Fig. 6. Observing the responses of Fig. 3 to Fig. 6, one can find that the closed-loop system is Lyapunov stable and it is passive in sense of Definition 1 via the fuzzy controller (60). Besides, from the simulated responses, one can obtain $\|u(k)\|=$ 


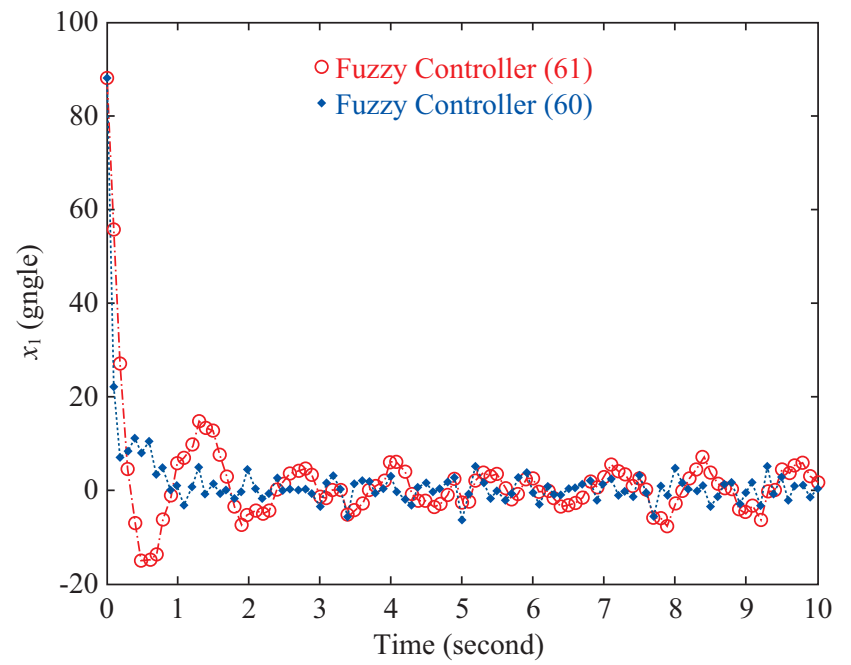

Fig. 7. Comparisons of fuzzy controllers Eqs. (60) and (61) for state $x_{1}(k)$.

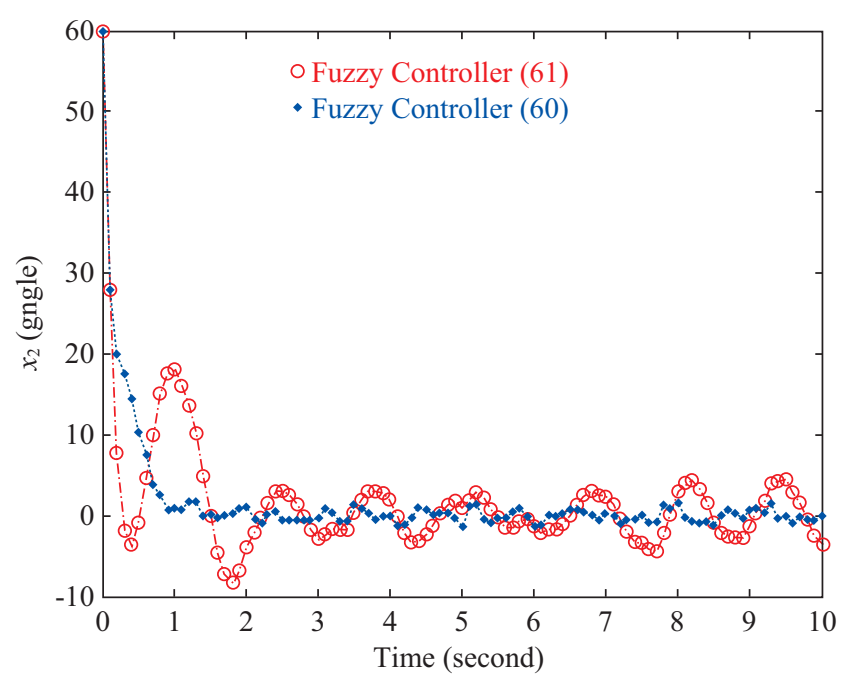

Fig. 8. Comparisons of fuzzy controllers Eqs. (60) and (61) for state $x_{2}(k)$.

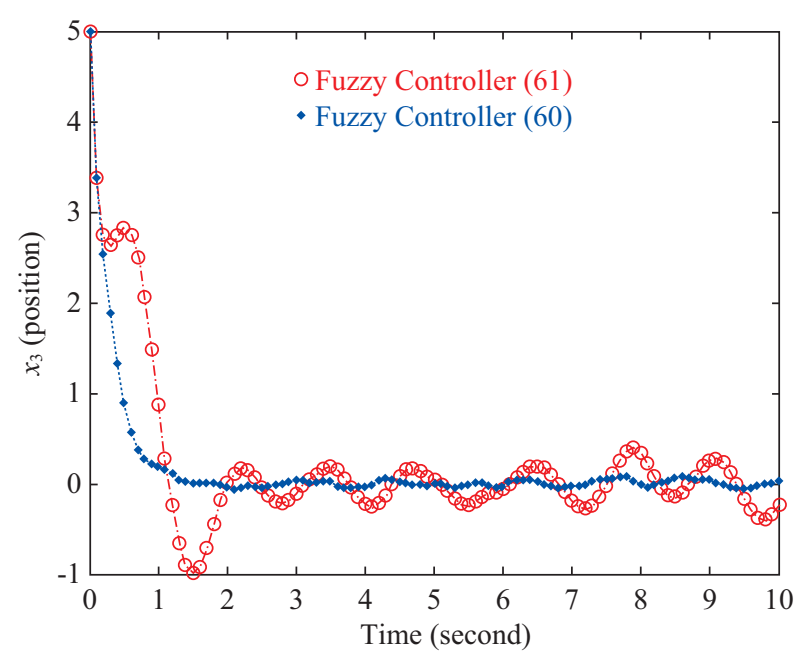

Fig. 9. Comparisons of fuzzy controllers Eqs. (60) and (61) for state $x_{3}(k)$.
2.6794 that is smaller than the desired input constraint (58), i.e., $\mu=2.7103$. Hence, the nonlinear system (47) can be achieved performance of input constraint via designed fuzzy controller (60).

In order to compare the proposed design method with previous fuzzy control method, the approach developed in [16] is applied to stabilize the same nonlinear system (47) with the same input constraint (58). Applying the design method of [16], one can obtain the following fuzzy controller.

$$
u_{h 1}(k)=\sum_{\mathrm{i}=1}^{\mathrm{r}} h_{\mathrm{i}}(x(k))\left(-\mathbf{F}_{\mathrm{i}} x(k)\right)
$$

where $\mathbf{F}_{1}=\left[\begin{array}{lll}-1.359 & 0.9222 & -0.0779\end{array}\right]$,

$\mathbf{F}_{2}=\left[\begin{array}{lll}-1.3434 & 0.7772 & -0.0969\end{array}\right]$,

$\mathbf{F}_{3}=\left[\begin{array}{lll}-1.359 & 0.9222 & -0.0779\end{array}\right]$. Employing the fuzzy controller (61) to control the system (47), the simulation results can be obtained with the same initial conditions $x(0)=$ $\left[\begin{array}{lll}88^{0} & 60^{\circ} & 5\end{array}\right]^{\mathrm{T}}$ in Fig. 7 to Fig. 9. From the responses, one can obtain $\left\|u_{h 1}(k)\right\|=2.3494$ that also achieves the desired input constraint. In conclusions, the input constraints of system (47) controlled by controllers (60) and (61) are both achieved. However, from the Fig. 7 to Fig. 9, one can find that the performances of setting time and overshoot of controller (60) is better than that of controller (61). Thus, the proposed fuzzy controller design method provides a better solution than the previous design approach developed in [16].

Without loss of generality, the performances of setting time and overshoot can be improved if the input constraint is not considered in the controller design. Thus, the fuzzy controller design method without considering input constraint [17] is applied in this example to compare with the proposed fuzzy control method. Applying the method of [17] to design the fuzzy controller, one can obtain the following fuzzy controller by solving some LMI stability conditions.

$$
u_{h 2}(k)=\sum_{\mathrm{i}=1}^{\mathrm{r}} h_{\mathrm{i}}(x(k))\left(-\mathbf{F}_{\mathrm{i}} x(k)\right)
$$

where $\mathbf{F}_{1}=\left[\begin{array}{lll}-3.205 & 4.1698 & -0.5899\end{array}\right]$,

$\mathbf{F}_{2}=\left[\begin{array}{lll}-3.0037 & 3.0136 & -0.5967\end{array}\right]$,

$\mathbf{F}_{3}=\left[\begin{array}{lll}-3.205 & 4.1698-0.5899\end{array}\right]$. The Fig. 10 to Fig. 12 show the simulation responses of system (47) that is controlled by the fuzzy controller (62). It can be found that the performances of setting time and overshoot of (62) are better than that of (61). They are similar to the performance responses of (60). However, from the simulation results, one can obtain $\left\|u_{h 2}(k)\right\|=4.6266$ that does not achieve the desired input constraint (58). Therefore, one can find that the proposed fuzzy control method can provide improvements for the previous works. 


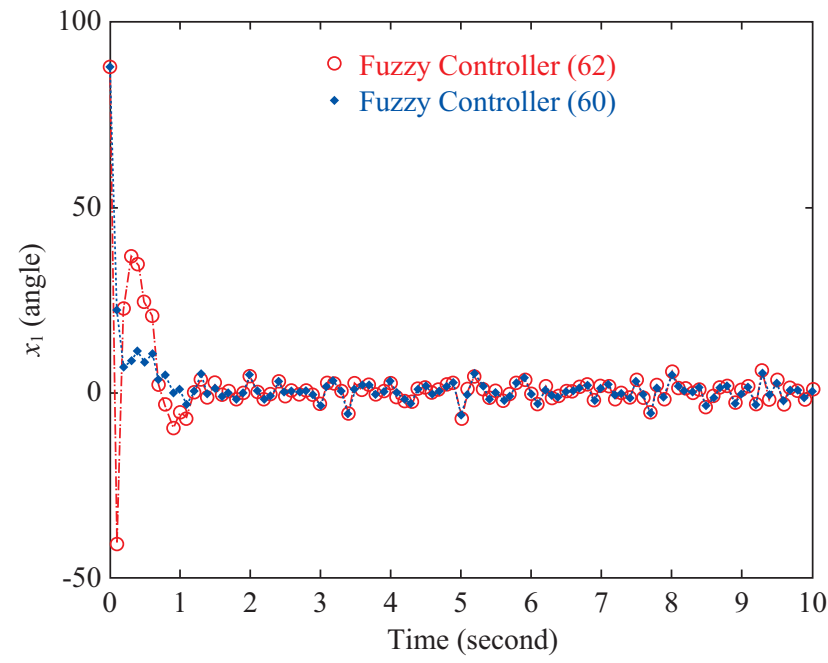

Fig. 10. Comparisons of fuzzy controllers Eqs. (60) and (62) for state $x_{1}(k)$.

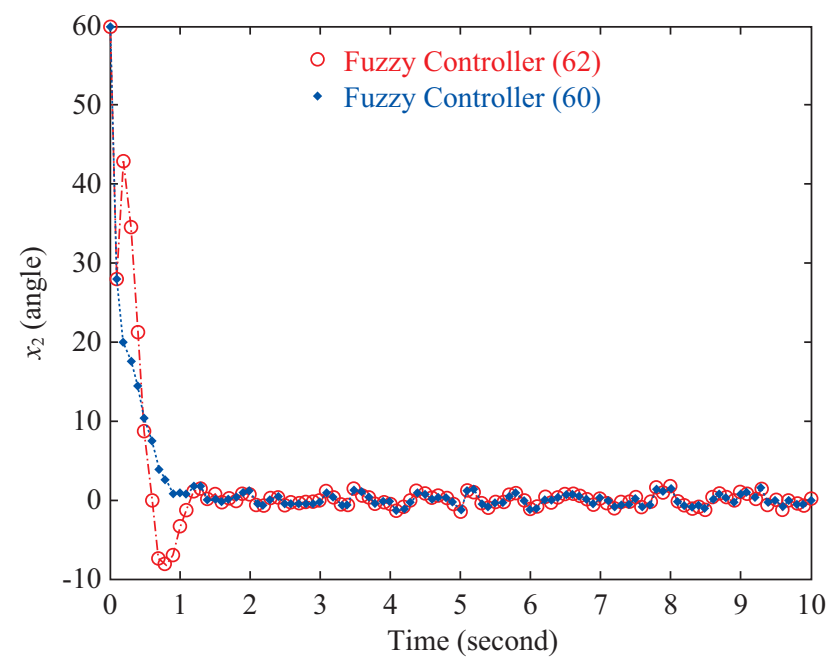

Fig. 11. Comparisons of fuzzy controllers Eqs. (60) and (62) for state $x_{2}(k)$.

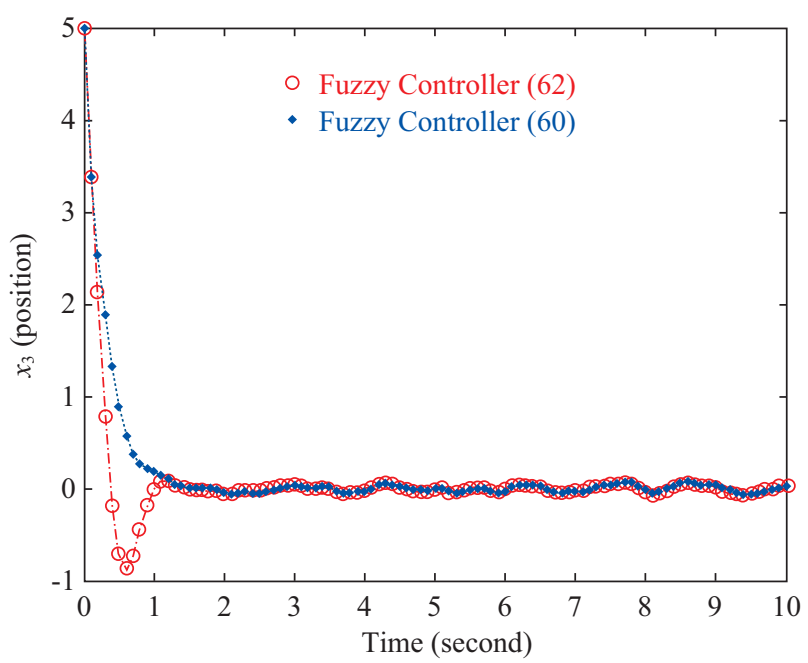

Fig. 12. Comparisons of fuzzy controllers Eqs. (60) and (62) for state $x_{3}(k)$.

\section{CONCLUSIONS}

Using the properties of the passivity and input constraint, this paper has discussed the stability analysis and controller design synthesis for the discrete-time affine T-S fuzzy models. The Lyapunov function associated with the stored function for passivity property was used to derive the stability conditions. The stability conditions were first derived of the BMI forms, which were transformed into LMI forms by applying the auxiliary variables in this paper. Moreover, a modified ILMI algorithm was developed to solve the above stability conditions and to obtain the feasible solutions of fuzzy controllers. For controlling a nonlinear truck-trailer system, a numerical example has been provided to demonstrate the effectiveness and applicability of the proposed fuzzy controller design approach.

\section{ACKNOWLEDGMENTS}

The authors would like to express their sincere gratitude to anonymous reviewers who gave us some constructive comments, criticisms and suggestions. This work was supported by the National Science Council of the Republic of China under Contract NSC97-2221-E-019-023.

\section{REFERENCES}

1. Boyd, S., Ghaoui, L. E., Feron, E., and Balakrishnan, V., Linear Matrix Inequalities in System and Control Theory, SIAM, Philadelphia, PA (1994).

2. Chang, W. J. and Chang, W., "Synthesis of nonlinear discrete control systems via time-delay affine Takagi-Sugeno fuzzy models," ISA Transactions, Vol. 44, No. 2, pp. 243-257 (2005).

3. Chang, W. J. and Chang, W., "Discrete fuzzy control of time-delay affine Takagi-Sugeno fuzzy models with $\mathrm{H}_{\infty}$ constraint," IEE Proceeding, Part D, Control Theory and Applications, Vol. 153, No. 6, pp. 745-752 (2006).

4. Chang, W. J., Ku, C. C., and Chang, W., "Analysis and synthesis of discrete nonlinear passive systems via affine T-S fuzzy Models," International Journal of Systems Science, Vol. 39, No. 8, pp. 809-821 (2008).

5. Chang, W. J. and Shing, C. C., "Discrete fuzzy covariance control for specified decay rate," International Journal of Systems Science, Vol. 36, No. 4, pp. 201-208 (2005).

6. Chang, W. J. and Sun, C. C., "Constrained fuzzy controller design of discrete Takagi-Sugeno fuzzy models,” Fuzzy Sets and Systems, Vol. 133, No. 1, pp. 37-55 (2003).

7. Chang, W. J. and Yeh, Y. L., "Observer-based fuzzy covariance control for discrete nonlinear systems," Journal of Marine Science and Technology, Vol. 15, No. 3, pp. 255-264 (2007).

8. Chu, H. Y., Tsai, K. H., and Chang, W. J., "Fuzzy control of active queue management routers for transmission control protocol networks via time-delay affine Takagi-Sugeno fuzzy models," International Journal of Innovative Computing, Information and Control, Vol. 4, No. 2, pp. 291312 (2008).

9. Fridman, E. and Shaked, U., "On delay-dependent passivity," IEEE Transactions on Automatic Control, Vol. 47, No. 4, pp. 664-669 (2002).

10. Kim, E. and Kim, D., "Stability analysis and synthesis for an affine fuzzy control system via LMI and ILMI: discrete case," IEEE Transactions on System, Man and Cybernetics, Vol. 31, No. 1, pp. 132-140 (2001).

11. Li, Z., Wang, J., and Shao, H., "Delay-dependent dissipative control for linear time-delay systems," Journal of the Franklin Institute, Vol. 339, No. 6, pp. 529-542 (2002). 
12. Lin, W. and Byrnes, C. I., "Passivity and absolute stabilization of a class of discrete-time nonlinear systems," Automatica, Vol. 31, No. 2, pp. $263-$ 267 (1995).

13. Lozano, R., Brogliato, B., Egeland, O., and Maschke, B., Dissipative Systems Analysis and Control: Theory and Application, Springer, London (2000).

14. Shao, H. and Feng, C., "Robustly quadratic dissipative analysis and control for multivariable linear discrete-time systems," Proceeding of IEEE Conference, Intelligent Control and Automation, Vol. 1, pp. 543547 (2004).

15. Tan, Z., Soh, Y. C., and Xie, L., "Dissipative control for linear discretetime systems," Automatica, Vol. 35, No. 9, pp. 1557-1564 (1999).

16. Tanaka, K., Ikeda, T., and Wang, H. O., "Fuzzy control system design via LMIs," Proceeding of American Control Conference, New Mexico, Vol. 5, pp. 2873-2877 (1997).
17. Tanaka, K. and Sano, M., “A robust stabilization problem of fuzzy control systems and its application to backing up control of a truck-trailer," IEEE Transactions on Fuzzy Systems, Vol. 2, No. 2, pp. 119-134 (1994).

18. Tanaka, K. and Wang, H. O., Fuzzy Control Systems Design and AnalysisA Linear Matrix Inequality Approach, John Wiley \& Sons, Inc., New York (2001).

19. Wang, H. O., Tanaka, K., and Griffin, M. F., "An approach to fuzzy control of nonlinear systems: stability and design issues," IEEE Transactions on Fuzzy Systems, Vol. 4, No. 1, pp. 13-23 (1996).

20. Willems, J. C. and Trentrlman, H. L., "Synthesis of dissipative systems using quadratic differential forms: part I," IEEE Transactions on Automatic Control, Vol. 47, No. 1, pp. 53-69 (2002).

21. Willems, J. C. and Trentrlman, H. L., "Synthesis of dissipative systems using quadratic differential forms: part II," IEEE Transactions on Automatic Control, Vol. 47, No. 1, pp. 70-86 (2002). 